\title{
Verticillium dahliae Populations from Mint and Potato Are Genetically Divergent with Predominant Haplotypes
}

\author{
Jeremiah K. S. Dung, Tobin L. Peever, and Dennis A. Johnson
}

Washington State University, Pullman 99164-6430.

Accepted for publication 22 October 2012.

\begin{abstract}
Dung, J. K. S., Peever, T. L., and Johnson D. A. 2013. Verticillium dahliae populations from mint and potato are genetically divergent with predominant haplotypes. Phytopathology 103:445-459.

In total, 286 Verticillium dahliae isolates from mint, potato, and other hosts and substrates were characterized for mating type, vegetative compatibility group (VCG), and multilocus microsatellite haplotype to determine population genetic structure among populations infecting mint and potato. Populations from mint and potato fit a clonal reproductive model, with all isolates a single mating type (MAT1-2) and multiple occurrences of the same haplotypes. Haplotype $\mathrm{H} 02$ represented $88 \%$ of mint isolates and was primarily VCG2B, while haplotype H04 repre-

$\mathrm{H} 02$ and H04 typically caused severe disease on mint and potato, respectively, in greenhouse assays regardless of host origin. Principal coordinate analysis and analysis of molecular variance indicated that mint and potato populations were significantly genetically diverged $(P=0.02)$, and identification of private alleles and estimation of migration rates suggested restricted gene flow. Migration was detected between infected potato plants and seed tubers, infested tare soil, and field soils. Genetic differentiation of $V$. dahliae from mint and potato may be due to the occurrence of a single mating type and differences in VCG. Populations of $V$. dahliae in potato and mint were characterized by the presence of aggressive, clonally reproducing haplotypes which are widely distributed in commercial mint and potato production.
\end{abstract} sented $70 \%$ of potato isolates and was primarily VCG4A. Haplotypes
Verticillium wilt is an economically significant disease of many dicotyledonous crops, including mint (Mentha L.) and potato (Solanum tuberosum L.). Mint and potato are important crops in the United States and especially in the Pacific Northwest (Idaho, Oregon, and Washington States). The Columbia Basin region is a semiarid, irrigated region located in Washington and Oregon states and is a leading producer of high-quality mint oil produced from peppermint, scotch spearmint, and native spearmint, with $>23,000$ ha under production in these two states in 2011. The area is also a leading grower of potato in the United States, with $>80,000$ ha harvested in 2011. In the Columbia Basin, mint and potato can be grown in close proximity or in rotation with each other. Verticillium wilt is a persistent problem in both mint and potato production in the region.

Verticillium dahliae (Kleb.), causal agent of Verticillium wilt, is a soilborne fungus with a wide host range that includes $>200$ dicotyledonous plants. The fungus can survive for many years in the soil in the form of microsclerotia, where it remains dormant until it germinates in response to root exudates. Despite its wide host range, $V$. dahliae exhibits a range of aggressiveness on different host plants, suggesting host specialization or host adaptation (8). Differences in aggressiveness among $V$. dahliae populations associated with mint and potato are well documented $(11,16,24,33,39)$.

The pathogen is only known to reproduce asexually, although mating type idiomorphs in a heterothallic arrangement have been described, which suggests that meiotic recombination may be possible (43). The presence of both mating types could potentially lead to sexual reproduction and the production of new sources of inoculum in the form of ascospores. Various vegetative compati-

Corresponding author: J. K. S. Dung;

E-mail address: jeremiah.dung@ oregonstate.edu

http://dx.doi.org/10.1094/PHYTO-06-12-0133-R

(C) 2013 The American Phytopathological Society bility groups (VCGs) exist in $V$. dahliae, whereby isolates are grouped based on the ability of nitrate-nonutilizing (nit) mutants to undergo hyphal anastomosis with known tester strains (23). VCGs are thought to limit gene flow among populations of $V$. dahliae and may contribute to genetic drift and differentiation (27). However, parasexual recombination can occur through hyphal anastomosis between like VCGs. Genetic recombination, through sexual, asexual, or a combination of both processes, can lead to greater genotypic diversity and a better ability to survive and respond to dynamic environments and control measures. The genetic and genotypic diversity of $V$. dahliae populations infecting different hosts is likely a result of the population diversity present in the soil and the presence or absence of hostadapted genotypes, the number of mating types and VCGs present, and the role of other sources of inoculum such as infected propagative materials.

Correlations between VCG subgroups and pathogenicity, phenotype, or geographic distributions have been observed and may be important in Verticillium wilt epidemiology (26). However, such correlations do not always occur and all known VCGs have the potential to infect a wide range of hosts under greenhouse conditions $(8,35)$. Isolates from mint are typically VCG2B and highly aggressive on mint, suggesting the presence of a mintadapted VCG2B pathotype $(11,16,30,35)$. In North America, VCG4A isolates of $V$. dahliae are primarily found associated with potato, followed by VCG4B and VCG2 $(24,33,39,40)$. Both VCG4 and VCG2 often cause the most severe symptoms on potato, and VCG4A isolates of $V$. dahliae from potato exhibit the greatest aggressiveness on potato. Strong host-specific interactions may exert high selection pressure on the pathogen, resulting in differentiation among pathogen populations by host and limiting the diversity of populations on mint compared with potato. This may be especially true for vascular wilt pathogens, which must successfully invade the vascular system of hosts to cause disease, form resting structures, and complete the disease cycle. The occurrence of highly aggressive VCG2B and VCG4A isolates 
from mint and potato may indicate the presence of specific populations with particular specialization on these hosts.

Knowledge regarding the genetic and genotypic diversity of $V$. dahliae infecting mint and potato will provide additional insights into the epidemiology and management of Verticillium wilt in these two important hosts. Microsatellites, or simple sequence repeats, are putatively neutral markers which are widely used to detect genetic and genotypic diversity in populations (4). Previous studies using microsatellite markers have detected genetic and genotypic differentiation among $V$. dahliae populations from different hosts and geographic regions $(3,7)$ and, in one study, recombination was detected among populations (3). However, information regarding the mating type and genetic diversity of $V$. dahliae isolates infecting mint and potato in North America is lacking. We hypothesize that populations of $V$. dahliae from mint will have reduced genotypic variability relative to populations from potato due to the more limited production of mint (7 U.S. states compared with 50 states for potato), the predominance of VCG2B on the host, and the occurrence of a host-adapted phenotype. In contrast, potato may exhibit higher levels of genotypic diversity than mint due to the occurrence of multiple VCGs and the wide-scale production of potato across North America.

This study placed a particular emphasis on $V$. dahliae populations impacting the Columbia Basin region of the U.S. Pacific Northwest. In this region, approximately half of the mint fields are grown in rotation with potato while $\approx 10 \%$ of potato fields are cropped in rotation with mint (32), and the potential exists for $V$. dahliae inoculum associated with mint production to subsequently cause Verticillium wilt in potato and vice versa. In addition, $>156,300$ metric tons of seed potato were planted in the Columbia Basin in 2007 (25), and infected seed tubers or infested tare soil associated with seed lots can provide potential sources of new strains of $V$. dahliae to be introduced into Columbia Basin fields $(13,33)$. The primary objective of this research was to quantify the genetic and genotypic diversity and differentiation among $V$. dahliae populations from mint and potato in North America using microsatellites, mating types, and VCG analysis. The genetic and genotypic diversity of $V$. dahliae isolates from infected seed tubers and infested soils associated with certified potato seed lots was also estimated because they represent a potential source of infection and infestation in commercial potato production. Isolates from field soils associated with potato production in Washington and Ohio were also included. Pathogenicity assays were performed in the greenhouse to determine the aggressiveness of various $V$. dahliae haplotypes on these two hosts.

\section{MATERIALS AND METHODS}

Fungal isolates. For this study, 286 isolates were used. Isolates were stored on sterile filter paper at $-20^{\circ} \mathrm{C}$ and maintained on $1 \%$ potato dextrose agar (potato dextrose agar at $10 \mathrm{~g} /$ liter and agar at $15 \mathrm{~g} / \mathrm{liter}$ ). The mint population comprised 104 isolates from infected mint, including peppermint $(n=74)$, Scotch spearmint $(n$ $=12)$, and native spearmint $(n=18)$. The potato population consisted of 119 isolates isolated from potato, certified seed potato, or soil associated with certified seed potato. Mint isolates were mostly sampled from the Columbia Basin of Washington in $1996(n=85)$. Isolates from mint in the Columbia Basin were obtained from 32 fields located in five counties. Up to three monoconidial isolates were obtained from different plants in each field. Additional isolates from other mint-producing areas of the United States (Idaho, Indiana, Michigan, Montana, and Oregon) and years (pre-1996, 2006, and 2007) were included for comparison with the Washington isolates. Twenty isolates of $V$. dahliae were sampled from infected potato plants in Idaho, North Dakota, New York, Ohio, Pennsylvania, Washington, and Wisconsin and represented a range of collection years (pre-1977 through 2010).
Isolates of $V$. dahliae were obtained from infected seed tubers produced in several states (Idaho, Maine, Michigan, Montana, Nebraska, New York, Oregon, Pennsylvania, South Dakota, Washington, and Wisconsin; $n=72$ ) in 1995, 1996, and 2007 to 2010, as well as from infested soil adhering to seed tubers (Idaho, Montana, North Dakota, and Oregon; $n=17$ ) and from bags and trucks used to transport certified seed lots $(n=10)$ between 2009 and 2010. $V$. dahliae isolates from field soils associated with potato production were also included $(n=20)$. V. dahliae isolates from other hosts and various states $(n=43)$ were included to provide an outgroup and represent the potential genetic and genotypic diversity of $V$. dahliae. Isolate designations, VCG, host and geographic origins, collection years, and sources are listed in Table 1. VCGs of the majority of isolates were determined by other workers or in previous studies $(10,11,23,32,33)$ but VCGs of 79 isolates were characterized in this study using reference strains provided by Dr. R. Rowe (Ohio Agricultural Research and Development Center, The Ohio State University) as previously described $(12,23)$.

Amplification of mating type idiomorphs. DNA was extracted from lyophilized mycelia using a glass bead disruption technique (10). The quantity and quality of DNA was measured using agarose gel electrophoresis and a NanoDrop 1000 spectrophotometer (Thermo Fisher Scientific, Wilmington, DE). Eluted DNA was stored at $-20^{\circ} \mathrm{C}$ until needed. Mating type of $V$. dahliae isolates was determined using a multiplex polymerase chain reaction (PCR) assay with primer pairs VdMAT1-1a/VdMAT1-1b and VdMAT1-2a/VdMAT1-2b (43). These primers amplify the MAT1-1 ( $\approx 600$ bp of product) and MAT1-2 ( $\approx 400 \mathrm{bp}$ of product) idiomorphs, respectively. PCR reaction mixtures included $10 \mathrm{ng}$ of $V$. dahliae DNA, 1× GoTaq Colorless Mastermix (Promega Corp., Madison, WI), and $200 \mathrm{nM}$ each primer in $10 \mu \mathrm{l}$ reactions. Amplifications were performed in Bio-Rad DNA Engine thermocyclers (Bio-Rad Laboratories, Hercules, CA), using the following cycling parameters: denaturing at $94^{\circ} \mathrm{C}$ for $3 \mathrm{~min}$, followed by 30 cycles of denaturing at $94^{\circ} \mathrm{C}$ for $30 \mathrm{~s}$, annealing at $65^{\circ} \mathrm{C}$ for $30 \mathrm{~s}$, and extension at $72^{\circ} \mathrm{C}$ for $1 \mathrm{~min}$. A final extension was carried out at $72^{\circ} \mathrm{C}$ for $3 \mathrm{~min}$. All reactions were repeated once. The PCR products were electrophoresed on a $2 \%$ (wt/vol) agarose gel containing $0.5 \times$ Tris-borate-EDTA and visualized using ethidium bromide.

Microsatellite amplification. Ten microsatellite markers (VD1, VD2, VD3, VD4, VD8, VD10, VD26, VD27, VD74, and VD96), which were previously developed and shown to be polymorphic in $V$. dahliae populations infecting other hosts $(2,3)$, were used in the study. Repeat motifs were 3 to $10 \mathrm{bp}$ in length and previously localized to four different chromosomes using the $V$. dahliae genome sequence of strain VdLs17, which can be found at the Verticillium Group Database (http://www.broad.mit. edu/annotation/genome/verticillium_dahliae/MultiHome.html) (2). Genotyping was performed using fluorescently labeled M13 primers as described by Schuelke (37), with modifications. PCR reagents included: $500 \mathrm{pg}$ of $V$. dahliae DNA, $1 \times$ GoTaq colorless PCR reaction buffer (Promega Corp.), $400 \mathrm{nM}$ dNTP mix, $2.5 \mathrm{mM} \mathrm{MgCl}$, $1.5 \mathrm{U}$ of GoTaq polymerase, $250 \mathrm{nM}$ reverse primer, $50 \mathrm{nM}$ forward primer amended at the $5^{\prime}$ end with the universal M13 primer sequence (CACGACGTTGTAAAACGAC), and $200 \mathrm{nM}$ universal M13 primer labeled with one of four fluorophores: FAM or HEX (Applied Biosystems, Foster City, CA), or ATTO 550 or ATTO 565 (ATTO-TEC, Siegen, Germany). Amplifications were performed independently for each locus in Bio-Rad DNA Engine thermocyclers (Bio-Rad Laboratories), using the following cycling parameters: denaturing at $95^{\circ} \mathrm{C}$ for $2 \mathrm{~min}$, followed by 35 cycles of denaturing at $95^{\circ} \mathrm{C}$ for $10 \mathrm{~s}$, annealing at $58^{\circ} \mathrm{C}$ for $10 \mathrm{~s}$, and extension at $72^{\circ} \mathrm{C}$ for $30 \mathrm{~s}$. A final extension was carried out at $72^{\circ} \mathrm{C}$ for $5 \mathrm{~min}$. Four differentially labeled PCR products were multiplexed for fragment analysis. Each labeled PCR product ( $3 \mu \mathrm{l})$ was combined in $13 \mu \mathrm{l}$ of sterile, 


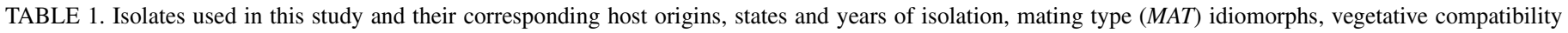
groups (VCG), multilocus haplotypes, and sources ${ }^{\mathrm{a}}$

\begin{tabular}{|c|c|c|c|c|c|c|c|}
\hline Sample group, isolate & Host & State $^{b}$ & Year & $\begin{array}{c}M A T \\
\text { idiomorph }\end{array}$ & VCG & $\begin{array}{l}\text { Multilocus } \\
\text { haplotype }\end{array}$ & Source \\
\hline \multicolumn{8}{|l|}{ Mint } \\
\hline 17 & Native spearmint & WA & 1996 & $M A T 1-2$ & $2 \mathrm{~B}$ & $\mathrm{H} 02$ & L. Douhan \\
\hline 19 & Native spearmint & WA & 1996 & $M A T 1-2$ & $2 \mathrm{~B}$ & $\mathrm{H} 02$ & L. Douhan \\
\hline 20 & Native spearmint & WA & 1996 & $M A T 1-2$ & $2 \mathrm{~A} / \mathrm{B}$ & $\mathrm{H} 02$ & L. Douhan \\
\hline 46 & Native spearmint & WA & 1996 & $M A T 1-2$ & $2 \mathrm{~B}$ & $\mathrm{H} 02$ & L. Douhan \\
\hline 47 & Native spearmint & WA & 1996 & MAT1-2 & $2 \mathrm{~B}$ & $\mathrm{H} 02$ & L. Douhan \\
\hline 48 & Native spearmint & WA & 1996 & $M A T 1-2$ & $2 \mathrm{~B}$ & $\mathrm{H} 02$ & L. Douhan \\
\hline 52 & Native spearmint & WA & 1996 & $M A T 1-2$ & $2 \mathrm{~B}$ & $\mathrm{H} 02$ & L. Douhan \\
\hline 53 & Native spearmint & WA & 1996 & $M A T 1-2$ & $2 \mathrm{~B}$ & H31 & L. Douhan \\
\hline 54 & Native spearmint & WA & 1996 & $M A T 1-2$ & $2 \mathrm{~B}$ & $\mathrm{H} 02$ & L. Douhan \\
\hline 55 & Native spearmint & WA & 1996 & $M A T 1-2$ & $2 \mathrm{~B}$ & $\mathrm{H} 02$ & L. Douhan \\
\hline 56 & Native spearmint & WA & 1996 & $M A T 1-2$ & $2 \mathrm{~B}$ & $\mathrm{H} 02$ & L. Douhan \\
\hline 57 & Native spearmint & WA & 1996 & $M A T 1-2$ & 2B & $\mathrm{H} 02$ & L. Douhan \\
\hline 73 & Native spearmint & WA & 1996 & $M A T 1-2$ & $2 \mathrm{~B}$ & $\mathrm{H} 02$ & L. Douhan \\
\hline 74 & Native spearmint & WA & 1996 & $M A T 1-2$ & $2 \mathrm{~B}$ & $\mathrm{H} 02$ & L. Douhan \\
\hline 103 & Native spearmint & WA & 1996 & $M A T 1-2$ & $2 \mathrm{~B}$ & $\mathrm{H} 02$ & L. Douhan \\
\hline 104 & Native spearmint & WA & 1996 & $M A T 1-2$ & $2 \mathrm{~B}$ & $\mathrm{H} 02$ & L. Douhan \\
\hline 121 & Native spearmint & WA & 1996 & $M A T 1-2$ & $2 \mathrm{~B}$ & $\mathrm{H} 02$ & L. Douhan \\
\hline 124 & Native spearmint & WA & 1996 & $M A T 1-2$ & $2 \mathrm{~B}$ & $\mathrm{H} 02$ & L. Douhan \\
\hline 152 & Peppermint & ID & 1996 & $M A T 1-2$ & $2 \mathrm{~B}$ & $\mathrm{H} 29$ & L. Douhan \\
\hline 155 & Peppermint & ID & 1996 & $M A T 1-2$ & $4 \mathrm{~A}$ & H04 & L. Douhan \\
\hline 13 & Peppermint & IN & 1996 & $M A T 1-2$ & $2 \mathrm{~B}$ & $\mathrm{H} 02$ & L. Douhan \\
\hline 8 & Peppermint & MI & 1996 & $M A T 1-2$ & $2 \mathrm{~B}$ & Н30 & L. Douhan \\
\hline 9 & Peppermint & MI & 1996 & $M A T 1-2$ & ND & $\mathrm{H} 02$ & L. Douhan \\
\hline 10 & Peppermint & MI & 1996 & $M A T 1-2$ & $2 \mathrm{~B}$ & $\mathrm{H} 02$ & L. Douhan \\
\hline 29 & Peppermint & MT & 1996 & MAT1-2 & $2 \mathrm{~B}$ & $\mathrm{H} 02$ & L. Douhan \\
\hline 134 & Peppermint & MT & 1996 & MAT1-2 & $2 \mathrm{~B}$ & $\mathrm{H} 02$ & L. Douhan \\
\hline 136 & Peppermint & MT & 1996 & $M A T 1-2$ & $2 \mathrm{~B}$ & $\mathrm{H} 02$ & L. Douhan \\
\hline 156 & Peppermint & MT & 1996 & MAT1-2 & $2 \mathrm{~B}$ & $\mathrm{H} 02$ & L. Douhan \\
\hline 150 & Peppermint & OR & 1996 & MAT1-2 & $2 \mathrm{~B}$ & $\mathrm{H} 02$ & L. Douhan \\
\hline 151 & Peppermint & OR & 1996 & MAT1-2 & $2 \mathrm{~B}$ & $\mathrm{H} 28$ & L. Douhan \\
\hline 157 & Peppermint & OR & 1996 & MAT1-2 & $2 \mathrm{~B}$ & $\mathrm{H} 02$ & L. Douhan \\
\hline 97-098-1 & Peppermint & OR & 1996 & MAT1-2 & $2 \mathrm{~B}$ & $\mathrm{H} 02$ & L. Douhan \\
\hline 1 & Peppermint & WA & 1996 & MAT1-2 & $2 \mathrm{~B}$ & $\mathrm{H} 02$ & L. Douhan \\
\hline 5 & Peppermint & WA & 1996 & $M A T 1-2$ & $2 \mathrm{~B}$ & $\mathrm{H} 02$ & L. Douhan \\
\hline 24 & Peppermint & WA & 1996 & MAT1-2 & $2 \mathrm{~B}$ & $\mathrm{H} 02$ & L. Douhan \\
\hline 25 & Peppermint & WA & 1996 & MAT1-2 & $2 \mathrm{~B}$ & $\mathrm{H} 02$ & L. Douhan \\
\hline 27 & Peppermint & WA & 1996 & MAT1-2 & $2 \mathrm{~B}$ & $\mathrm{H} 02$ & L. Douhan \\
\hline 32 & Peppermint & WA & 1996 & $M A T 1-2$ & $2 \mathrm{~B}$ & $\mathrm{H} 02$ & L. Douhan \\
\hline 34 & Peppermint & WA & 1996 & MAT1-2 & $2 \mathrm{~B}$ & $\mathrm{H} 02$ & L. Douhan \\
\hline 35 & Peppermint & WA & 1996 & MAT1-2 & $2 \mathrm{~B}$ & $\mathrm{H} 02$ & L. Douhan \\
\hline 36 & Peppermint & WA & 1996 & MAT1-2 & $2 \mathrm{~B}$ & $\mathrm{H} 02$ & L. Douhan \\
\hline 37 & Peppermint & WA & 1996 & MAT1-2 & $2 \mathrm{~B}$ & $\mathrm{H} 02$ & L. Douhan \\
\hline 38 & Peppermint & WA & 1996 & $M A T 1-2$ & $2 \mathrm{~B}$ & $\mathrm{H} 02$ & L. Douhan \\
\hline 41 & Peppermint & WA & 1996 & $M A T 1-2$ & $2 \mathrm{~B}$ & $\mathrm{H} 02$ & L. Douhan \\
\hline 43 & Peppermint & WA & 1996 & MAT1-2 & $2 \mathrm{~B}$ & $\mathrm{H} 02$ & L. Douhan \\
\hline 44 & Peppermint & WA & 1996 & $M A T 1-2$ & $2 \mathrm{~B}$ & $\mathrm{H} 02$ & L. Douhan \\
\hline 45 & Peppermint & WA & 1996 & MAT1-2 & $2 \mathrm{~B}$ & $\mathrm{H} 02$ & L. Douhan \\
\hline 49 & Peppermint & WA & 1996 & $M A T 1-2$ & $2 \mathrm{~B}$ & $\mathrm{H} 02$ & L. Douhan \\
\hline 50 & Peppermint & WA & 1996 & $M A T 1-2$ & $2 \mathrm{~B}$ & $\mathrm{H} 02$ & L. Douhan \\
\hline 51 & Peppermint & WA & 1996 & $M A T 1-2$ & $2 \mathrm{~B}$ & $\mathrm{H} 02$ & L. Douhan \\
\hline 58 & Peppermint & WA & 1996 & $M A T 1-2$ & $2 \mathrm{~B}$ & $\mathrm{H} 02$ & L. Douhan \\
\hline 60 & Peppermint & WA & 1996 & $M A T 1-2$ & $2 \mathrm{~B}$ & $\mathrm{H} 02$ & L. Douhan \\
\hline 61 & Peppermint & WA & 1996 & $M A T 1-2$ & $2 \mathrm{~B}$ & $\mathrm{H} 02$ & L. Douhan \\
\hline 62 & Peppermint & WA & 1996 & $M A T 1-2$ & $2 \mathrm{~B}$ & $\mathrm{H} 02$ & L. Douhan \\
\hline 65 & Peppermint & WA & 1996 & $M A T 1-2$ & $2 \mathrm{~B}$ & $\mathrm{H} 02$ & L. Douhan \\
\hline 67 & Peppermint & WA & 1996 & $M A T 1-2$ & $2 \mathrm{~B}$ & $\mathrm{H} 02$ & L. Douhan \\
\hline 68 & Peppermint & WA & 1996 & $M A T 1-2$ & $2 \mathrm{~B}$ & $\mathrm{H} 02$ & L. Douhan \\
\hline 69 & Peppermint & WA & 1996 & $M A T 1-2$ & $2 \mathrm{~B}$ & $\mathrm{H} 02$ & L. Douhan \\
\hline 70 & Peppermint & WA & 1996 & $M A T 1-2$ & $2 \mathrm{~B}$ & $\mathrm{H} 02$ & L. Douhan \\
\hline 71 & Peppermint & WA & 1996 & $M A T 1-2$ & $2 \mathrm{~B}$ & $\mathrm{H} 02$ & L. Douhan \\
\hline 72 & Peppermint & WA & 1996 & $M A T 1-2$ & $2 \mathrm{~B}$ & $\mathrm{H} 02$ & L. Douhan \\
\hline 77 & Peppermint & WA & 1996 & $M A T 1-2$ & $2 \mathrm{~B}$ & $\mathrm{H} 02$ & L. Douhan \\
\hline 78 & Peppermint & WA & 1996 & $M A T 1-2$ & $2 \mathrm{~B}$ & $\mathrm{H} 02$ & L. Douhan \\
\hline \multirow[t]{2}{*}{79} & Peppermint & WA & 1996 & $M A T 1-2$ & $2 \mathrm{~B}$ & H02 & L. Douhan \\
\hline & & & & & & \multicolumn{2}{|c|}{ (continued on next page) } \\
\hline
\end{tabular}

${ }^{a} \mathrm{ND}=$ not determined and Unk = unknown.

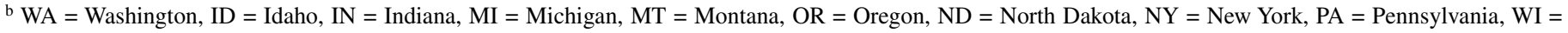
Wisconsin, $\mathrm{OH}=$ Ohio, $\mathrm{SD}=$ South Dakota, $\mathrm{ME}=$ Maine, $\mathrm{N} / \mathrm{A}=$ not applicable, $\mathrm{AZ}=$ Arizona, $\mathrm{CA}=\mathrm{California}$, and $\mathrm{TX}=\mathrm{Texas}$.

c Isolate was obtained from the same seed lot as the isolate above.

d Previously characterized as VCG2B but characterized as VCG4B in this study.

e Previously characterized as VCG2B but characterized as VCG4A in this study. 


\begin{tabular}{|c|c|c|c|c|c|c|c|}
\hline Sample group, isolate & Host & State $^{b}$ & Year & $\begin{array}{c}M A T \\
\text { idiomorph }\end{array}$ & $\mathrm{VCG}$ & $\begin{array}{l}\text { Multilocus } \\
\text { haplotype }\end{array}$ & Source \\
\hline 80 & Peppermint & WA & 1996 & $M A T 1-2$ & $2 \mathrm{~B}$ & $\mathrm{H} 02$ & L. Douhan \\
\hline 82 & Peppermint & WA & 1996 & $M A T 1-2$ & $2 \mathrm{~B}$ & $\mathrm{H} 30$ & L. Douhan \\
\hline 84 & Peppermint & WA & 1996 & $M A T 1-2$ & $2 \mathrm{~B}$ & $\mathrm{H} 02$ & L. Douhan \\
\hline 92 & Peppermint & WA & 1996 & $M A T 1-2$ & ND & $\mathrm{H} 02$ & L. Douhan \\
\hline 93 & Peppermint & WA & 1996 & MAT1-2 & $2 \mathrm{~B}$ & $\mathrm{H} 02$ & L. Douhan \\
\hline 94 & Peppermint & WA & 1996 & $M A T 1-2$ & $2 \mathrm{~B}$ & $\mathrm{H} 02$ & L. Douhan \\
\hline 95 & Peppermint & WA & 1996 & MAT1-2 & $2 \mathrm{~B}$ & $\mathrm{H} 02$ & L. Douhan \\
\hline 96 & Peppermint & WA & 1996 & $M A T 1-2$ & $2 \mathrm{~B}$ & $\mathrm{H} 02$ & L. Douhan \\
\hline 97 & Peppermint & WA & 1996 & MAT1-2 & $2 \mathrm{~B}$ & $\mathrm{H} 02$ & L. Douhan \\
\hline 101 & Peppermint & WA & 1996 & $M A T 1-2$ & $2 \mathrm{~B}$ & H02 & L. Douhan \\
\hline 102 & Peppermint & WA & 1996 & $M A T 1-2$ & $2 \mathrm{~B}$ & $\mathrm{H} 02$ & L. Douhan \\
\hline 106 & Peppermint & WA & 1996 & $M A T 1-2$ & $2 \mathrm{~B}$ & $\mathrm{H} 02$ & L. Douhan \\
\hline 107 & Peppermint & WA & 1996 & $M A T 1-2$ & $2 \mathrm{~B}$ & $\mathrm{H} 02$ & L. Douhan \\
\hline 109 & Peppermint & WA & 1996 & $M A T 1-2$ & $2 \mathrm{~B}$ & $\mathrm{H} 02$ & L. Douhan \\
\hline 111 & Peppermint & WA & 1996 & $M A T 1-2$ & $2 \mathrm{~B}$ & $\mathrm{H} 02$ & L. Douhan \\
\hline 112 & Peppermint & WA & 1996 & MAT1-2 & $2 \mathrm{~B}$ & $\mathrm{H} 02$ & L. Douhan \\
\hline 113 & Peppermint & WA & 1996 & $M A T 1-2$ & $2 \mathrm{~B}$ & $\mathrm{H} 02$ & L. Douhan \\
\hline 114 & Peppermint & WA & 1996 & $M A T 1-2$ & $2 \mathrm{~B}$ & $\mathrm{H} 02$ & L. Douhan \\
\hline 115 & Peppermint & WA & 1996 & $M A T 1-2$ & $2 \mathrm{~B}$ & $\mathrm{H} 02$ & L. Douhan \\
\hline 116 & Peppermint & WA & 1996 & $M A T 1-2$ & $2 \mathrm{~B}$ & $\mathrm{H} 02$ & L. Douhan \\
\hline 119 & Peppermint & WA & 1996 & MAT1-2 & $2 \mathrm{~B}$ & $\mathrm{H} 02$ & L. Douhan \\
\hline 128 & Peppermint & WA & 1996 & MAT1-2 & $2 \mathrm{~B}$ & $\mathrm{H} 02$ & L. Douhan \\
\hline 140 & Peppermint & WA & 1996 & MAT1-2 & $2 \mathrm{~B}$ & $\mathrm{H} 02$ & L. Douhan \\
\hline 141 & Peppermint & WA & 1996 & $M A T 1-2$ & $2 \mathrm{~B}$ & $\mathrm{H} 02$ & L. Douhan \\
\hline 142 & Peppermint & WA & 1996 & MAT1-2 & $2 \mathrm{~B}$ & $\mathrm{H} 02$ & L. Douhan \\
\hline Bi.Gr. & Peppermint & MT & pre-1997 & MAT1-2 & ND & $\mathrm{H} 02$ & T. Davis \\
\hline $\mathrm{V}-16$ & Peppermint & IN & 1991 & $M A T 1-2$ & $2 \mathrm{~B}$ & H33 & R. Green \\
\hline M.pi-06 & Peppermint & WA & 2006 & $M A T 1-2$ & $2 \mathrm{~B}$ & $\mathrm{H} 02$ & J. Dung \\
\hline 15 & Scotch spearmint & IN & pre-1996 & MAT1-2 & $2 \mathrm{~B}$ & $\mathrm{H} 27$ & L. Douhan \\
\hline 21 & Scotch spearmint & WA & 1996 & MAT1-2 & $2 \mathrm{~B}$ & H30 & L. Douhan \\
\hline 22 & Scotch spearmint & WA & 1996 & $M A T 1-2$ & $2 \mathrm{~B}$ & $\mathrm{H} 02$ & L. Douhan \\
\hline 66 & Scotch spearmint & WA & 1996 & $M A T 1-2$ & $2 \mathrm{~B}$ & $\mathrm{H} 02$ & L. Douhan \\
\hline 98 & Scotch spearmint & WA & 1996 & $M A T 1-2$ & $2 \mathrm{~B}$ & $\mathrm{H} 02$ & L. Douhan \\
\hline 105 & Scotch spearmint & WA & 1996 & $M A T 1-2$ & $2 \mathrm{~B}$ & $\mathrm{H} 02$ & L. Douhan \\
\hline 126 & Scotch spearmint & WA & 1996 & $M A T 1-2$ & $2 \mathrm{~B}$ & H35 & L. Douhan \\
\hline 127 & Scotch spearmint & WA & 1996 & $M A T 1-2$ & ND & $\mathrm{H} 02$ & L. Douhan \\
\hline 129 & Scotch spearmint & WA & 1996 & MAT1-2 & $2 \mathrm{~B}$ & H34 & L. Douhan \\
\hline 130 & Scotch spearmint & WA & 1996 & $M A T 1-2$ & $2 \mathrm{~B}$ & H34 & L. Douhan \\
\hline 145 & Scotch spearmint & WA & 1996 & MAT1-2 & $2 \mathrm{~B}$ & $\mathrm{H} 02$ & L. Douhan \\
\hline M.gr-07 & Scotch spearmint & WA & 2007 & $M A T 1-2$ & $2 \mathrm{~B}$ & $\mathrm{H} 02$ & J. Dung \\
\hline \multicolumn{8}{|l|}{ Potato } \\
\hline 240 & Potato plant & WA & pre-1977 & MAT1-2 & $4 \mathrm{~B}$ & $\mathrm{H} 21$ & J. Davis \\
\hline $\mathrm{V}-25$ & Potato plant & ID & pre-1983 & $M A T 1-2$ & $4 \mathrm{~A}$ & $\mathrm{H} 04$ & L. Sorenson \\
\hline 317 & Potato plant & ND & pre-1983 & MAT1-2 & $4 \mathrm{~A} / \mathrm{B}$ & H04 & G. Secor \\
\hline 318 & Potato plant & ND & pre-1983 & MAT1-2 & $4 \mathrm{~A}$ & H04 & G. Secor \\
\hline V-9-86 & Potato plant & NY & pre-1983 & $M A T 1-2$ & $4 \mathrm{~A} / \mathrm{B}$ & H09 & V. Stockwell \\
\hline V-13-86 & Potato plant & $\mathrm{PA}$ & pre-1983 & $M A T 1-2$ & $2 \mathrm{~A} / \mathrm{B}$ & $\mathrm{H} 26$ & V. Stockwell \\
\hline Par-4 & Potato plant & WI & 1983 & $M A T 1-2$ & $4 \mathrm{~B}$ & H09 & R. Rowe \\
\hline P-3 & Potato plant & $\mathrm{OH}$ & 1984 & MAT1-2 & $4 \mathrm{~B}$ & H08 & R. Rowe \\
\hline P-7 & Potato plant & $\mathrm{OH}$ & 1984 & $M A T 1-2$ & $4 \mathrm{~A}$ & $\mathrm{H} 22$ & R. Rowe \\
\hline AF $10-7$ & Potato plant & ID & pre-1994 & MAT1-2 & $4 \mathrm{~B}$ & H07 & C. Strausbaugh \\
\hline AF $2-3$ & Potato plant & ID & pre-1994 & MAT1-2 & $4 \mathrm{~A}$ & H04 & C. Strausbaugh \\
\hline 235 & Potato plant & WA & 1995 & MAT1-2 & $4 \mathrm{~B}$ & H08 & R. Rowe \\
\hline 239 & Potato plant & WA & 1995 & MAT1-2 & $4 \mathrm{~B}$ & $\mathrm{H} 07$ & R. Rowe \\
\hline 274 & Potato plant & WA & 1995 & MAT1-2 & $4 \mathrm{~B}$ & H08 & R. Rowe \\
\hline Vd.St-1 & Potato plant & ID & pre-1997 & MAT1-2 & $4 \mathrm{~A}$ & H04 & W. Schnathorst \\
\hline 233 & Potato plant & Unk & pre-1997 & MAT1-2 & $4 \mathrm{~A}$ & H11 & R. Rowe \\
\hline W.1721.2009 & Potato plant & WA & 2009 & MAT1-2 & $4 \mathrm{~A}$ & H04 & J. Dung \\
\hline TSPS.1.2010 & Potato plant & WA & 2010 & MAT1-2 & $4 \mathrm{~A}$ & H04 & J. Dung \\
\hline TSPS.2.2010 & Potato plant & WA & 2010 & MAT1-2 & $4 \mathrm{~A}$ & H04 & J. Dung \\
\hline VSP 712 & Potato plant & WA & Unk & MAT1-2 & $2 \mathrm{~B}$ & $\mathrm{H} 25$ & L. du Toit \\
\hline 653 & Seed tuber & ID & 1995 & $M A T 1-2$ & $4 \mathrm{~A}$ & $\mathrm{H} 04$ & R. Rowe \\
\hline W-83 & Seed tuber & MT & 1995 & $M A T 1-2$ & $4 \mathrm{~A}$ & H04 & R. Rowe \\
\hline W-201 & Seed tuber & MT & 1995 & $M A T 1-2$ & $4 \mathrm{~B}$ & H08 & R. Rowe \\
\hline W-152 & Seed tuber & OR & 1995 & MAT1-2 & $4 \mathrm{~B}$ & H13 & R. Rowe \\
\hline W-90 & Seed tuber & $\mathrm{SD}$ & 1995 & $M A T 1-2$ & $4 \mathrm{~A}$ & $\mathrm{H} 04$ & R. Rowe \\
\hline $\mathrm{W}-140$ & Seed tuber & WA & 1995 & $M A T 1-2$ & $4 \mathrm{~B}$ & H16 & R. Rowe \\
\hline W-87 & Seed tuber & WA & 1995 & $M A T 1-2$ & $4 \mathrm{~A}$ & H05 & R. Rowe \\
\hline W-100 & Seed tuber & WY & 1995 & MAT1-2 & $4 \mathrm{~A}$ & $\mathrm{H} 04$ & R. Rowe \\
\hline $11-11$ & Seed tuber & $\mathrm{ME}$ & 1996 & MAT1-2 & $4 \mathrm{~B}$ & H06 & R. Rowe \\
\hline $66-12$ & Seed tuber & $\mathrm{ME}$ & 1996 & $M A T 1-2$ & $4 \mathrm{~A}$ & $\mathrm{H} 20$ & R. Rowe \\
\hline $21-18$ & Seed tuber & MI & 1996 & $M A T 1-2$ & $4 \mathrm{~A}$ & H06 & R. Rowe \\
\hline \multirow[t]{2}{*}{$30-6$} & Seed tuber & $\mathrm{NE}$ & 1996 & MAT1-2 & $4 \mathrm{~A}$ & H04 & R. Rowe \\
\hline & & & & & & \multicolumn{2}{|c|}{ (continued on next page } \\
\hline
\end{tabular}


TABLE 1. (continued from preceding page)

\begin{tabular}{|c|c|c|c|c|c|c|c|}
\hline Sample group, isolate & Host & State $^{b}$ & Year & $\begin{array}{c}M A T \\
\text { idiomorph }\end{array}$ & VCG & $\begin{array}{l}\text { Multilocus } \\
\text { haplotype }\end{array}$ & Source \\
\hline $99-1$ & Seed tuber & NY & 1996 & MAT1-2 & $4 \mathrm{~A} / \mathrm{B}$ & H09 & R. Rowe \\
\hline $101-1$ & Seed tuber & NY & 1996 & $M A T 1-2$ & $4 \mathrm{~B}$ & H06 & R. Rowe \\
\hline $102-1$ & Seed tuber & NY & 1996 & $M A T 1-2$ & $4 \mathrm{~B}$ & H06 & R. Rowe \\
\hline $1-3$ & Seed tuber & $\mathrm{PA}$ & 1996 & MAT1-2 & $4 \mathrm{~A}$ & H08 & R. Rowe \\
\hline $83-1$ & Seed tuber & WI & 1996 & MAT1-2 & $4 \mathrm{~A}$ & H11 & R. Rowe \\
\hline S.15 & Seed tuber & ID & 2007 & MAT1-2 & $4 \mathrm{~A}$ & H04 & J. Dung \\
\hline SC.19 & Seed tuber & ID & 2007 & $M A T 1-2$ & $4 \mathrm{~A}$ & $\mathrm{H} 04$ & J. Dung \\
\hline TN.26 & Seed tuber & ID & 2007 & MAT1-2 & $4 \mathrm{~A}$ & H04 & J. Dung \\
\hline $\mathrm{TN} .40^{\mathrm{c}}$ & Seed tuber & ID & 2007 & MAT1-2 & $4 \mathrm{~A}$ & H04 & J. Dung \\
\hline TN.66 & Seed tuber & ID & 2007 & MAT1-2 & $4 \mathrm{~A}$ & H04 & J. Dung \\
\hline L7.T31 & Seed tuber & ID & 2008 & MAT1-2 & $4 \mathrm{~A}$ & H04 & J. Dung \\
\hline L8.T25 & Seed tuber & ID & 2008 & $M A T 1-2$ & $4 \mathrm{~A}$ & $\mathrm{H} 04$ & J. Dung \\
\hline L10.T15 & Seed tuber & MT & 2008 & MAT1-2 & $4 \mathrm{~A}$ & H04 & J. Dung \\
\hline $\mathrm{L} 10 . \mathrm{T} 17^{\mathrm{c}}$ & Seed tuber & MT & 2008 & $M A T 1-2$ & $4 \mathrm{~A}$ & $\mathrm{H} 04$ & J. Dung \\
\hline L10.T26 & Seed tuber & MT & 2008 & MAT1-2 & $4 \mathrm{~A}$ & H04 & J. Dung \\
\hline $\mathrm{L} 10 . \mathrm{T} 35^{\mathrm{c}}$ & Seed tuber & MT & 2008 & $M A T 1-2$ & $4 \mathrm{~A}$ & $\mathrm{H} 04$ & J. Dung \\
\hline $\mathrm{L} 10 . \mathrm{T}^{\mathrm{c}}$ & Seed tuber & MT & 2008 & MAT1-2 & $4 \mathrm{~A}$ & $\mathrm{H} 04$ & J. Dung \\
\hline L9.T12 & Seed tuber & MT & 2008 & $M A T 1-2$ & $4 \mathrm{~A}$ & H04 & J. Dung \\
\hline L2.T20 & Seed tuber & Unk & 2008 & MAT1-2 & ND & H08 & J. Dung \\
\hline L3.T19 & Seed tuber & Unk & 2008 & $M A T 1-2$ & $4 \mathrm{~A}$ & H04 & J. Dung \\
\hline 09-29.6.A & Seed tuber & ID & 2009 & $M A T 1-2$ & $4 \mathrm{~A}$ & $\mathrm{H} 04$ & J. Dung \\
\hline $09-41.15 .5$ & Seed tuber & ID & 2009 & MAT1-2 & $4 \mathrm{~A}$ & H04 & J. Dung \\
\hline $09-41.8 .3^{\mathrm{c}}$ & Seed tuber & ID & 2009 & $M A T 1-2$ & $4 \mathrm{~A}$ & $\mathrm{H} 04$ & J. Dung \\
\hline B.A-296 & Seed tuber & ID & 2009 & $M A T 1-2$ & $4 \mathrm{~A}$ & H04 & J. Dung \\
\hline L7.2009 & Seed tuber & ID & 2009 & MAT1-2 & $4 \mathrm{~B}$ & $\mathrm{H} 07$ & J. Dung \\
\hline $09-29.3$ & Seed tuber & MT & 2009 & $M A T 1-2$ & $4 \mathrm{~A}$ & $\mathrm{H} 04$ & J. Dung \\
\hline $09-29.9^{c}$ & Seed tuber & MT & 2009 & MAT1-2 & $4 \mathrm{~A}$ & H04 & J. Dung \\
\hline $09-54.5 \mathrm{C}$ & Seed tuber & MT & 2009 & $M A T 1-2$ & $4 \mathrm{~A}$ & H04 & J. Dung \\
\hline $09-55.8$ & Seed tuber & MT & 2009 & MAT1-2 & $4 \mathrm{~A}$ & H04 & J. Dung \\
\hline NG1.9 & Seed tuber & MT & 2009 & MAT1-2 & $4 \mathrm{~A}$ & $\mathrm{H} 04$ & J. Dung \\
\hline S.1-Umat-15.09 & Seed tuber & WA & 2009 & MAT1-2 & $4 \mathrm{~A}$ & H04 & J. Dung \\
\hline UG4.11 & Seed tuber & WA & 2009 & MAT1-2 & $4 \mathrm{~A}$ & $\mathrm{H} 04$ & J. Dung \\
\hline $\mathrm{UG} 4.16^{\mathrm{c}}$ & Seed tuber & WA & 2009 & MAT1-2 & $4 \mathrm{~A} / \mathrm{B}$ & $\mathrm{H} 04$ & J. Dung \\
\hline $\mathrm{UG} 4.18^{\mathrm{c}}$ & Seed tuber & WA & 2009 & $M A T 1-2$ & $4 \mathrm{~A}$ & H04 & J. Dung \\
\hline LS8-A & Seed tuber & WI & 2009 & $M A T 1-2$ & $4 \mathrm{~B}$ & $\mathrm{H} 07$ & J. Dung \\
\hline LS8-B ${ }^{c}$ & Seed tuber & WI & 2009 & $M A T 1-2$ & $4 \mathrm{~A}$ & H09 & J. Dung \\
\hline 12.A. 2010 & Seed tuber & ID & 2010 & MAT1-2 & $4 \mathrm{~A}$ & H04 & J. Dung \\
\hline 59.A.2010 & Seed tuber & ID & 2010 & MAT1-2 & $4 \mathrm{~A}$ & $\mathrm{H} 04$ & J. Dung \\
\hline 30.A.2010 & Seed tuber & MT & 2010 & MAT1-2 & $4 \mathrm{~A}$ & H04 & J. Dung \\
\hline 30. B. $2010^{c}$ & Seed tuber & MT & 2010 & MAT1-2 & $4 \mathrm{~A}$ & H04 & J. Dung \\
\hline 48.A.2010 & Seed tuber & MT & 2010 & MAT1-2 & $4 \mathrm{~A}$ & H04 & J. Dung \\
\hline 48.B. $2010^{c}$ & Seed tuber & MT & 2010 & $M A T 1-2$ & $4 \mathrm{~A}$ & $\mathrm{H} 04$ & J. Dung \\
\hline 52.A.2010 & Seed tuber & MT & 2010 & MAT1-2 & $4 \mathrm{~A}$ & $\mathrm{H} 04$ & J. Dung \\
\hline 52.C. $2010^{c}$ & Seed tuber & MT & 2010 & MAT1-2 & $4 \mathrm{~A}$ & H04 & J. Dung \\
\hline 20.C. 2010 & Seed tuber & OR & 2010 & MAT1-2 & $4 \mathrm{~A}$ & $\mathrm{H} 04$ & J. Dung \\
\hline 20.D. $2010^{c}$ & Seed tuber & OR & 2010 & $M A T 1-2$ & $4 \mathrm{~A}$ & $\mathrm{H} 23$ & J. Dung \\
\hline 20.F. $2010^{c}$ & Seed tuber & OR & 2010 & $M A T 1-2$ & $4 \mathrm{~B}$ & H15 & J. Dung \\
\hline 21.C. 2010 & Seed tuber & OR & 2010 & MAT1-2 & $4 \mathrm{~A}$ & H04 & J. Dung \\
\hline 21.D. $2010^{c}$ & Seed tuber & OR & 2010 & MAT1-2 & $4 \mathrm{~A}$ & H05 & J. Dung \\
\hline 21.F. $2010^{c}$ & Seed tuber & OR & 2010 & MAT1-2 & $4 \mathrm{~A}$ & H05 & J. Dung \\
\hline 21.H.2010 & Seed tuber & OR & 2010 & MAT1-2 & $4 \mathrm{~A}$ & H04 & J. Dung \\
\hline 21.I.2010 & Seed tuber & OR & 2010 & MAT1-2 & $4 \mathrm{~A}$ & H04 & J. Dung \\
\hline 29.A.2010 & Seed tuber & OR & 2010 & MAT1-2 & $4 \mathrm{~B}$ & H09 & J. Dung \\
\hline 38.A. 2010 & Seed tuber & OR & 2010 & MAT1-2 & $4 \mathrm{~A}$ & $\mathrm{H} 04$ & J. Dung \\
\hline 38. B. $2010^{c}$ & Seed tuber & OR & 2010 & $M A T 1-2$ & $4 \mathrm{~A}$ & $\mathrm{H} 04$ & J. Dung \\
\hline 38.C. $2010^{c}$ & Seed tuber & OR & 2010 & $M A T 1-2$ & $4 \mathrm{~B}$ & H14 & J. Dung \\
\hline 38.J.2010 & Seed tuber & OR & 2010 & MAT1-2 & $4 \mathrm{~A}$ & $\mathrm{H} 04$ & J. Dung \\
\hline 49.B. 2010 & Seed tuber & OR & 2010 & MAT1-2 & $4 \mathrm{~B}$ & H07 & J. Dung \\
\hline 55.A. 2010 & Seed tuber & Unk & 2010 & MAT1-2 & $4 \mathrm{~A}$ & $\mathrm{H} 04$ & J. Dung \\
\hline 3.1 .2010 & Seed tuber & Unk & 2010 & MAT1-2 & $4 \mathrm{~A}$ & H04 & J. Dung \\
\hline $09-41.8 .1$ & Seed tuber soil & ID & 2009 & MAT1-2 & $4 \mathrm{~A}$ & $\mathrm{H} 04$ & J. Dung \\
\hline $09-41.8 .2^{c}$ & Seed tuber soil & ID & 2009 & MAT1-2 & $4 \mathrm{~A}$ & H04 & J. Dung \\
\hline $09-45.2009$ & Seed tuber soil & ID & 2009 & MAT1-2 & $4 \mathrm{~A}$ & H19 & J. Dung \\
\hline $09-55.7 .1$ & Seed tuber soil & ID & 2009 & $M A T 1-2$ & $4 \mathrm{~A}$ & $\mathrm{H} 04$ & J. Dung \\
\hline $09-55.7 .2^{\mathrm{c}}$ & Seed tuber soil & ID & 2009 & MAT1-2 & $4 \mathrm{~A}$ & H04 & J. Dung \\
\hline Col.8 & Seed tuber soil & ID & 2009 & MAT1-2 & $4 \mathrm{~A}$ & H04 & J. Dung \\
\hline 09-48A.3.1 & Seed tuber soil & Unk & 2009 & MAT1-2 & $4 \mathrm{~A}$ & $\mathrm{H} 04$ & J. Dung \\
\hline 18.A.2010 & Seed tuber soil & ID & 2010 & $M A T 1-2$ & $4 \mathrm{~A}$ & $\mathrm{H} 04$ & J. Dung \\
\hline 18.B. $2010^{c}$ & Seed tuber soil & ID & 2010 & MAT1-2 & $4 \mathrm{~A}$ & H04 & J. Dung \\
\hline 28.2010 & Seed tuber soil & ID & 2010 & $M A T 1-2$ & $4 \mathrm{~A} / \mathrm{B}$ & $\mathrm{H} 04$ & J. Dung \\
\hline 19.2010 & Seed tuber soil & MT & 2010 & $M A T 1-2$ & $4 \mathrm{~A}$ & $\mathrm{H} 04$ & J. Dung \\
\hline 23.2010 & Seed tuber soil & MT & 2010 & $M A T 1-2$ & $4 \mathrm{~A}$ & $\mathrm{H} 04$ & J. Dung \\
\hline 44.A. 2010 & Seed tuber soil & MT & 2010 & MAT1-2 & $4 \mathrm{~A}$ & $\mathrm{H} 04$ & J. Dung \\
\hline
\end{tabular}


TABLE 1. (continued from preceding page)

\begin{tabular}{|c|c|c|c|c|c|c|c|}
\hline Sample group, isolate & Host & State $^{\mathrm{b}}$ & Year & $\begin{array}{c}M A T \\
\text { idiomorph }\end{array}$ & VCG & $\begin{array}{l}\text { Multilocus } \\
\text { haplotype }\end{array}$ & Source \\
\hline 57.A.2010 & Seed tuber soil & ND & 2010 & $M A T 1-2$ & $4 \mathrm{~A}$ & H04 & J. Dung \\
\hline 41.A.2010 & Seed tuber soil & OR & 2010 & $M A T 1-2$ & $4 \mathrm{~A}$ & H04 & J. Dung \\
\hline 6.A.2010 & Seed tuber soil & Unk & 2010 & $M A T 1-2$ & $4 \mathrm{~A}$ & H04 & J. Dung \\
\hline 6. B. $2010^{c}$ & Seed tuber soil & Unk & 2010 & $M A T 1-2$ & $4 \mathrm{~A}$ & H04 & J. Dung \\
\hline 103.Tare & Loose tare soil & N/A & 2010 & MAT1-2 & $4 \mathrm{~A}$ & H04 & J. Dung \\
\hline 105.Tare & Loose tare soil & N/A & 2010 & $M A T 1-2$ & $4 \mathrm{~A}$ & H04 & J. Dung \\
\hline 107.Tare & Loose tare soil & N/A & 2010 & $M A T 1-2$ & $4 \mathrm{~A}$ & $\mathrm{H} 04$ & J. Dung \\
\hline 111.Tare & Loose tare soil & N/A & 2010 & $M A T 1-2$ & $4 \mathrm{~A}$ & H04 & J. Dung \\
\hline 208.Tare & Loose tare soil & N/A & 2010 & $M A T 1-2$ & $4 \mathrm{~A}$ & H04 & J. Dung \\
\hline 212 Tare & Loose tare soil & N/A & 2010 & $M A T 1-2$ & $4 \mathrm{~A}$ & H04 & J. Dung \\
\hline 401 Tare & Loose tare soil & N/A & 2010 & $M A T 1-2$ & $4 \mathrm{~A}$ & H04 & J. Dung \\
\hline 402. Tare & Loose tare soil & N/A & 2010 & MAT1-2 & $4 \mathrm{~A}$ & H04 & J. Dung \\
\hline 404.Tare & Loose tare soil & N/A & 2010 & $M A T 1-2$ & $4 \mathrm{~A}$ & H04 & J. Dung \\
\hline 406.Tare & Loose tare soil & N/A & 2010 & MAT1-2 & $4 \mathrm{~A}$ & H04 & J. Dung \\
\hline \multicolumn{8}{|l|}{ Field soil } \\
\hline S-180 & Soil & $\mathrm{OH}$ & 1984 & $M A T 1-2$ & $4 \mathrm{~A} / \mathrm{B}$ & H06 & R. Rowe \\
\hline S-221 & Soil & $\mathrm{OH}$ & 1984 & $M A T 1-2$ & $2 \mathrm{~B}$ & H32 & R. Rowe \\
\hline S-39 & Soil & $\mathrm{OH}$ & 1984 & $M A T 1-2$ & $4 \mathrm{~B}$ & H12 & R. Rowe \\
\hline S-55 & Soil & $\mathrm{OH}$ & 1984 & $M A T 1-2$ & $4 \mathrm{~A}$ & H04 & R. Rowe \\
\hline S-80 & Soil & $\mathrm{OH}$ & 1984 & $M A T 1-2$ & $2 \mathrm{~B}$ & H32 & R. Rowe \\
\hline CB 51-4 & Soil & OR & 1998 & $M A T 1-2$ & $4 \mathrm{~A}$ & H04 & D. Johnson \\
\hline CB 65-2 & Soil & OR & 1998 & $M A T 1-2$ & $4 \mathrm{~B}$ & H08 & D. Johnson \\
\hline CB 1-2 & Soil & WA & 1998 & $M A T 1-2$ & $4 \mathrm{~A}$ & H04 & D. Johnson \\
\hline CB 18-1 & Soil & WA & 1998 & $M A T 1-2$ & $4 \mathrm{~B}$ & $\mathrm{H} 10$ & D. Johnson \\
\hline CB 19-2 & Soil & WA & 1998 & $M A T 1-2$ & $4 \mathrm{~A}$ & H04 & D. Johnson \\
\hline CB 22-4 & Soil & WA & 1998 & $M A T 1-2$ & $4 \mathrm{~A}$ & H04 & D. Johnson \\
\hline CB 2-3 & Soil & WA & 1998 & $M A T 1-2$ & $4 \mathrm{~A}$ & H04 & D. Johnson \\
\hline CB 27-3 & Soil & WA & 1998 & $M A T 1-2$ & $4 \mathrm{~A}$ & H04 & D. Johnson \\
\hline CB 4-2 & Soil & WA & 1998 & $M A T 1-2$ & $4 \mathrm{~A}$ & $\mathrm{H} 18$ & D. Johnson \\
\hline CB 4-6 & Soil & WA & 1998 & $M A T 1-2$ & $4 \mathrm{~B}$ & H08 & D. Johnson \\
\hline CB 71-1 & Soil & WA & 1998 & $M A T 1-2$ & $4 \mathrm{~A}$ & H04 & D. Johnson \\
\hline CB 72-3 & Soil & WA & 1998 & $M A T 1-2$ & $4 \mathrm{~A}$ & H04 & D. Johnson \\
\hline CB 74-4 & Soil & WA & 1998 & $M A T 1-2$ & $4 \mathrm{~A}$ & H04 & D. Johnson \\
\hline CB 76-1 & Soil & WA & 1998 & $M A T 1-2$ & $4 \mathrm{~A}$ & H04 & D. Johnson \\
\hline CB 86-7 & Soil & WA & 1998 & $M A T 1-2$ & $4 \mathrm{~A}$ & H04 & D. Johnson \\
\hline \multicolumn{8}{|l|}{ Other hosts } \\
\hline Vd-Agastache & Hyssop & Unk & Unk & MAT1-2 & 3 & $\mathrm{H} 45$ & D. Johnson \\
\hline Ash & Ash & Unk & Unk & $M A T 1-2$ & $4 \mathrm{~B}$ & H08 & P. Hamm \\
\hline 49-08 & Blackberry & OR & 2008 & $M A T 1-2$ & $4 \mathrm{~A}$ & $\mathrm{H} 04$ & J. Weiland \\
\hline 08-139 & Blackberry & WA & Unk & $M A T 1-2$ & $4 \mathrm{~A}$ & H04 & P. Hamm \\
\hline 601 & Cherry & WA & pre-1997 & $M A T 1-2$ & $4 \mathrm{~A}$ & H04 & D. Johnson \\
\hline DX-2 & Cotton & $\mathrm{AZ}$ & pre-1983 & $M A T 1-2$ & 1 & H01 & S. Alcorn \\
\hline T9 & Cotton & $\mathrm{CA}$ & pre-1983 & $M A T 1-2$ & 1 & H01 & P. Nicot \\
\hline $\mathrm{V}-44$ & Cotton & $\mathrm{TX}$ & pre-1983 & $M A T 1-2$ & 1 & H01 & J. Puhalla \\
\hline V-EMS(481) & Elm & $\mathrm{OH}$ & pre-1984 & $M A T 1-2$ & 1 & $\mathrm{H} 44$ & L. Schreiber \\
\hline $01-83$ & Maple & OR & 1983 & $M A T 1-2$ & $2 \mathrm{~B}$ & $\mathrm{H} 02$ & J. Weiland \\
\hline $06-166$ & Maple & Unk & Unk & $M A T 1-2$ & $4 \mathrm{~A}$ & H04 & P. Hamm \\
\hline $182-08$ & Maple & OR & 2008 & $M A T 1-2$ & $4 \mathrm{~A}$ & H41 & J. Weiland \\
\hline PCW & Pepper & $\mathrm{CA}$ & pre-1983 & $M A T 1-2$ & 3 & H03 & J. Puhalla \\
\hline Ca.35 & Pepper & $\mathrm{CA}$ & 1996 & $M A T 1-2$ & ND & Н36 & K. Subbarao \\
\hline $\mathrm{PH}$ & Pistachio & $\mathrm{CA}$ & pre-1983 & $M A T 1-2$ & $2 \mathrm{~A}$ & $\mathrm{H} 40$ & J. Puhalla \\
\hline $08-128$ & Black raspberry & WA & Unk & $M A T 1-2$ & $4 \mathrm{~A}$ & H04 & P. Hamm \\
\hline $08-139$ & Red raspberry & WA & Unk & $M A T 1-2$ & $4 \mathrm{~A}$ & H04 & P. Hamm \\
\hline VSP 08-33 A3 & Skullcap & WA & 2008 & $M A T 1-2$ & ND & $\mathrm{H} 02$ & L. du Toit \\
\hline VSP 08-33 C4 & Skullcap & WA & 2008 & $M A T 1-2$ & ND & $\mathrm{H} 02$ & L. du Toit \\
\hline $09-123.1$ & Skullcap & WA & 2009 & $M A T 1-2$ & $2 \mathrm{~B}$ & $\mathrm{H} 02$ & L. du Toit \\
\hline $09-123.2$ & Skullcap & WA & 2009 & $M A T 1-2$ & $2 \mathrm{~B}$ & $\mathrm{H} 02$ & L. du Toit \\
\hline $09-123.3$ & Skullcap & WA & 2009 & $M A T 1-2$ & $2 \mathrm{~B}$ & $\mathrm{H} 02$ & L. du Toit \\
\hline $09-123.4$ & Skullcap & WA & 2009 & $M A T 1-2$ & ND & $\mathrm{H} 02$ & L. du Toit \\
\hline $09-123.5$ & Skullcap & WA & 2009 & $M A T 1-2$ & ND & $\mathrm{H} 02$ & L. du Toit \\
\hline $09-123.6$ & Skullcap & WA & 2009 & $M A T 1-2$ & $2 \mathrm{~B}$ & $\mathrm{H} 02$ & L. du Toit \\
\hline SL-2 & Skullcap & WA & 2009 & $M A T 1-2$ & ND & $\mathrm{H} 02$ & J. Dung \\
\hline SL-3 & Skullcap & WA & 2009 & $M A T 1-2$ & $2 \mathrm{~B}$ & $\mathrm{H} 02$ & J. Dung \\
\hline VD1 VSP695 & Spinach & WA & 2001 & $M A T 1-2$ & $2 \mathrm{~B}$ & $\mathrm{H} 42$ & L. du Toit \\
\hline VD5 VSP699 & Spinach & WA & 2001 & $M A T 1-2$ & $4 \mathrm{~B}^{\mathrm{d}}$ & H07 & L. du Toit \\
\hline VD4 VSP698 & Spinach & WA & 2002 & $M A T 1-2$ & $4 \mathrm{~B}$ & H39 & L. du Toit \\
\hline VSP263 & Spinach & WA & 2003 & $M A T 1-2$ & $4 B$ & H07 & L. du Toit \\
\hline VSP268 & Spinach & WA & 2003 & $M A T 1-2$ & $4 \mathrm{~A}^{\mathrm{e}}$ & H07 & L. du Toit \\
\hline VSP678 & Spinach & WA & 2004 & MAT1-2 & $2 \mathrm{~B}$ & $\mathrm{H} 43$ & L. du Toit \\
\hline Fca. 29 & Strawberry & $\mathrm{CA}$ & 1996 & $M A T 1-2$ & $4 \mathrm{~B}$ & H17 & K. Subbarao \\
\hline F608 & Sugar beet & ID & 2007 & $M A T 1-2$ & $4 \mathrm{~A}$ & H04 & C. Strausbaugh \\
\hline F611 & Sugar beet & ID & 2007 & $M A T 1-2$ & $4 \mathrm{~A}$ & H04 & C. Strausbaugh \\
\hline F612 & Sugar beet & ID & 2007 & $M A T 1-2$ & $4 \mathrm{~A}$ & H04 & C. Strausbaugh \\
\hline
\end{tabular}


TABLE 1. (continued from preceding page)

\begin{tabular}{|c|c|c|c|c|c|c|c|}
\hline Sample group, isolate & Host & State $^{\mathrm{b}}$ & Year & $\begin{array}{c}M A T \\
\text { idiomorph }\end{array}$ & VCG & $\begin{array}{l}\text { Multilocus } \\
\text { haplotype }\end{array}$ & Source \\
\hline F616 & Sugar beet & ID & 2007 & MAT1-2 & $4 \mathrm{~A}$ & H04 & C. Strausbaugh \\
\hline F625 & Sugar beet & ID & 2007 & $M A T 1-2$ & $2 \mathrm{~B}$ & $\mathrm{H} 02$ & C. Strausbaugh \\
\hline 461 & Tomato & $\mathrm{OH}$ & 1984 & $M A T 1-1$ & 2 & Н37 & R. Rowe \\
\hline VMD-4 & Tomato & NY & pre-1983 & $M A T 1-2$ & $2 \mathrm{~A} / \mathrm{B}$ & Н38 & M. Lacy \\
\hline $97-147-2$ & Watermelon & OR & Unk & $M A T 1-2$ & $4 \mathrm{~A}$ & H04 & P. Hamm \\
\hline 381 & Watermelon & $\mathrm{OH}$ & 1981 & $M A T 1-2$ & $2 \mathrm{~A} / \mathrm{B}$ & $\mathrm{H} 24$ & S. Miller \\
\hline
\end{tabular}

distilled water for a total volume of $25 \mu \mathrm{l}$. In total, $3 \mu \mathrm{l}$ of the resulting mixture of amplicons was combined with $10 \mu \mathrm{l}$ of Hi-Di formamide and $0.03 \mu \mathrm{l}$ of LIZ-665 size standard (Applied Biosystems), and denatured at $95^{\circ} \mathrm{C}$ for $5 \mathrm{~min}$. Sizes of the fluorescently labeled fragments were determined using capillary electrophoresis on an ABI 3730xl DNA Analyzer (Applied Biosystems) at the United States Department of Agriculture Wheat Genetics, Quality, Physiology, and Disease Research Lab in Pullman, WA. Fragment analysis was performed in GeneMarker (SoftGenetics LLC, State College, PA). All PCR reactions and fragment analyses were independently replicated using the same DNA extractions to assess data reproducibility. In addition, a subset of isolates representing all 45 multilocus haplotypes observed in this study were genotyped using independently grown cultures, DNA extracts, PCR reactions, and fragment analyses.

Assessment of homoplasy among microsatellite loci. Representative fragments of each size class amplified for each locus were sequenced to verify allelism and provide an assessment of homoplasy in the data set (18). All putative alleles were sequenced at least once but, if an allele accounted for $\geq 10 \%$ of the observed loci, attempts were made to sequence the allele from at least four different isolates from various hosts and geographic locations. Sequencing was performed in $25 \mu \mathrm{l}$ reactions consisting of $200 \mu \mathrm{M}$ dNTPs, $2.5 \mathrm{mM} \mathrm{MgCl} 2,200 \mathrm{nM}$ each primer, $1 \mathrm{U}$ of GoTaq polymerase (Promega Corp.), and $10 \mathrm{ng}$ of template DNA in 1× GoTaq buffer under the PCR conditions described above. The quality and quantity of DNA was determined using a NanoDrop 1000 spectrophotometer and by gel electrophoresis. Two 3- $\mu$ l aliquots of each PCR product were treated with ExoSAP-IT (USB Corporation, Cleveland), prepared as previously described (12), and sequenced in both directions by Elim Biopharmaceuticals, Inc. (Hayward, CA).

Estimates of genetic diversity and genotypic richness, evenness, and diversity. Nei's expected heterozygosity $\left(H_{\text {exp }}\right)$, which is equivalent to genetic diversity in haploid organisms and defined as the probability that two randomly selected haplotypes are different (29), was calculated using the software Arlequin, ver. 3.5 (19). $H_{\exp }$ was also calculated for each locus in Arlequin using full and clone-corrected data sets. Heterozygosity values from mint and potato sample groups were compared using the MannWhitney U test. Rarefaction curves were estimated in Multilocus, ver. $1.3 \mathrm{~b}$ to estimate mean genotypic diversity expected in relation to the number of loci analyzed using 1,000 random samples (1). The observed number of genotypes $\left(g_{\text {obs }}\right)$ was obtained based on the total number of unique multilocus haplotypes observed in each sample group. The number of haplotypes expected in each sample group after correcting for the smallest sample size $\left(g_{\text {exp }}\right)$ was calculated using 10,000 jackknife replicates in GenoDive, ver. 1.1 (28). Multilocus genotypic diversity was estimated using Shannon-Wiener's $H^{\prime}$, Stoddart and Taylor's $G$, and the index $N_{1}$, while multilocus genotypic evenness was estimated using the indices $G / g_{\text {obs }}, E_{1}$, and $E_{5}$. Indices of multilocus genotypic diversity, evenness, and $95 \%$ confidence intervals were calculated using a modified $<$ jackboot $>$ macro and 2,000 bootstrap replicates in SAS (version 9.2; SAS Institute, Cary, NC), with the exception of $G / g_{\text {obs }}(21)$. Genotypic indices were considered significantly different if there were no overlaps of $95 \%$ confidence intervals.
Subsequent analyses were conducted using clone-corrected data and default software parameters under the stepwise mutation model, unless otherwise noted (31).

Genetic differentiation between populations from mint and potato. A matrix of genetic distances between multilocus haplotypes was estimated in GenoType (28). Pairwise genetic distances were plotted using principal coordinate analysis (PCoA) in GeneAlex, ver. 6.41 (34) to visualize genetic clusters among isolates from different sample groups and VCGs. Minimum spanning networks of VCG2B isolates from mint and VCG4 isolates from potato were estimated from the genetic distance matrix using MINSPNET (20), plotted in HapStar, ver. 0.5 (42), and visualized using the open-source vector graphics editor Inkscape, ver. 0.48 (http://www.inkscape.org).

Slatkin's $R_{\mathrm{st}}$ was estimated (38) and pairwise comparisons of isolates collected from infected potato plants, infected certified seed tubers, and infested tare soil from certified seed lots were performed to determine whether isolates associated with potato could be grouped together. Pairwise comparisons of populations from mint, potato, other hosts, and infested field soils associated with commercial potato production were also conducted. All pairwise comparisons were performed using the sequential Bonferroni method (36) and 16,000 permutations in Arlequin. Analysis of molecular variance (AMOVA) was performed to test the null hypothesis that $V$. dahliae populations associated with mint and potato were not genetically differentiated. AMOVA was also conducted to test the null hypothesis that VCG4A and VCG4B populations of $V$. dahliae from potato are not significantly differentiated. AMOVA and pairwise comparisons of Slatkin's $R_{\mathrm{st}}$ were performed in Arlequin using full and clone-corrected sets with 16,000 permutations.

Private alleles and migration rate estimates. The number of private alleles, scaled for the smallest clone-corrected population size $(n=9)$, was estimated for VCG2B haplotypes from mint and VCG4A and VCG4B haplotypes from potato using rarefaction curves in ADZE, ver. 1.0 (41). The program Migrate, ver. 3.0 $(5,6)$, was used to calculate maximum likelihood estimations of the mutation-scaled population size $(\theta)$ and mutation-scaled migration rates $(M)$ among isolates associated with potato (plants, seed tubers, tare soil, and field soils). Migration rates between VCG2B haplotypes from mint and VCG4A and VCG4B haplotypes from potato were also determined. Five runs were performed using a continuous Brownian motion model and heating applied at four temperatures $(1.0,1.5,3.0$, and 9.0). The run which resulted in the smallest likelihood value is reported. Five short chains, consisting of 1,000 samples sampled at increments of 20, were performed and 1,000 trees were recorded. Five long chains were performed with 5,000 samples, a burn-in of 10,000, and 5,000 trees were recorded.

Aggressiveness assays. Eighteen isolates were selected for aggressiveness assays on mint and potato based on multilocus haplotype, VCG, and host origin. Mint and potato assays were performed separately and repeated once. All treatments were replicated four times and blocked in the greenhouse. Experiments were arranged in the greenhouse as a randomized complete block design. Inoculum, consisting of a conidial suspension $\left(10^{6} \mathrm{CFU} / \mathrm{ml}\right)$, was prepared as previously described (16). A 
VCG2B isolate from mint (isolate 111) and a VCG4A isolate from potato (isolate 653) were included as positive controls. Isolates 111 and 653 were previously shown to be pathogenic on mint (16) and potato $(11,15)$, respectively, and were characterized according to multilocus haplotype in this study. Water-inoculated negative controls were included in all experiments.

Scotch spearmint (Mentha $\times$ gracilis) cuttings were rooted in LC1 potting mix for 4 weeks prior to inoculation. Plants were inoculated using a soil drench of conidial suspension to a final concentration of $10^{5} \mathrm{CFU} / \mathrm{cm}^{3}$ soil. Verticillium wilt symptoms were evaluated weekly beginning at 4 weeks post inoculation until 8 weeks postinoculation, using the following disease severity index (DSI): $0=$ no visible symptoms; $1=$ mild chlorosis $<10 \%$ of plant; 2 = distinct chlorosis 10 to $25 \%$ of plant; $3=$ asymmetrical apical growth, chlorosis 25 to $50 \%$ of plant or stunting ( $<75 \%$ height of control plants); $4=$ chlorosis $\geq 50 \%$ of plant or severe stunting $(<50 \%$ height of control plants); $5=$ necrosis $\geq 50 \%$ of plant; and $6=$ dead or nearly dead plant. DSI ratings were converted to area under the disease progress curve (AUDPC) using the formula $\sum_{i}^{n-1}\left[\left(Y_{i}+Y_{i+1}\right) / 2\right]\left(t_{i+1}-t_{i}\right)$, where $Y_{i}=$ disease rating at the $i$ th observation, $t_{i}=$ time (days post inoculation) at the $i$ th observation, and $n=$ number of observations.

Certified seed tubers ('Russet Norkotah') were cut into pieces $(\approx 57 \mathrm{~g})$ and planted into 3.8 -liter pots containing LC1 potting mix. After 7 weeks, plants were inoculated as described above. Total chlorosis and necrosis were recorded at 4, 5, and 6 weeks post inoculation and values were combined into areas under senescence progress curves (AUSPC) using the formula $\sum_{i}^{n-1}\left[\left(Y_{i}+\right.\right.$ $\left.\left.Y_{i+1}\right) / 2\right]\left(t_{i+1}-t_{i}\right)$, where $Y_{i}=$ cumulative senescence (percent chlorosis and percent necrosis) at the $i$ th observation, $t_{i}=$ time (days post inoculation) at the $i$ th observation, and $n=$ number of observations.

Analysis of variance was conducted on AUDPC and AUSPC data in SAS using PROC MIXED. Data were analyzed as a oneway factorial design with trials and blocks as random effects. Multiple pairwise comparisons against the water-inoculated control treatments were performed using Dunnett's test (17).

\section{RESULTS}

Mating type analysis. All but one isolate amplified a 400-bp band in the $M A T$-specific PCR assay and were designated as mating type $M A T 1-2$. The only exception was isolate 461 from tomato, which amplified a 600-bp band and was designated as MAT1-1.

Assessment of homoplasy among microsatellite loci. Sequence analysis of representative fragments of each size class amplified from each locus confirmed that similar-sized fragments were allelic. Differences in fragment sizes were primarily due to expected expansions and contractions in microsatellite repeat motifs. Sequences of VD4 alleles were only obtained from three to four isolates due to the repeated difficulty in obtaining usable sequences from this locus. Sequence analysis confirmed that small differences in fragment sizes ( 1 to $2 \mathrm{bp}$ ) detected using fragment analysis were caused by insertions or deletions in the microsatellite flanking regions but these did not affect interpretation of alleles. Potential compound microsatellites were observed in VD26 [(GCAGAGAG $\left.\left.{ }_{\mathrm{n} 1}\right)\left(\mathrm{GCACAGAG}_{\mathrm{n} 2}\right)\right]$ and VD27 $\left[\left(\mathrm{CAATGCCTCG}_{\mathrm{n} 1}\right)\left(\mathrm{CCATGCCTCG}_{\mathrm{n} 2}\right)\right]$. Because the inclusion of these polymorphisms did not result in the identification of any new haplotypes, they were also disregarded in the analyses. In addition, homoplasy was identified in two VD1 (CCTG) alleles which had identical fragment sizes but possessed one to three single-nucleotide polymorphisms that were either $\mathrm{G}-\mathrm{C}$ or $\mathrm{C}-\mathrm{G}$ transversions or G-A transitions. The transversions (G-C) and transitions (G-A) observed in VD1 resulted in the loss or gain of CCTG repeats, and slightly different results could be obtained depending on the method of scoring (fragment analysis versus sequence analyses). For consistency, results from fragment analysis were used for marker VD1. Furthermore, these polymorphisms were not included in the analyses due to the relatively small number of potentially homoplasious alleles (1.4\% of loci analyzed), the large amount of variability observed over all 10 loci, and the difficulty in incorporating such data with microsatellites into an appropriate mutation model (18).

Estimates of genetic diversity and genotypic richness, evenness, and diversity. Genetic diversity, based on Nei's heterozygosity $\left(H_{\text {exp }}\right)(29)$, was greater among the potato population $\left(H_{\exp }=0.51\right)$ compared with the mint population $\left(H_{\exp }=0.22\right)$ (Table 2) but this difference was not significant $(P=0.70)$. The potato population also had greater $H_{\exp }$ values compared with the mint population at the allelic level (Table 3) but the differences were also not significant using either full $(P=0.10)$ or clonecorrected $(P=0.21)$ data sets. Within the potato population, isolates from potato plants exhibited the greatest gene diversity $\left(H_{\exp }=0.83\right)$, followed by isolates from potato seed tubers $\left(H_{\exp }=\right.$ $0.53)$ and seed tuber soil $\left(H_{\exp }=0.12\right)$. Only one multilocus haplotype was observed among isolates obtained from seed lot soil. Among isolates from mint, $V$. dahliae isolates from native spearmint exhibited the least gene diversity $\left(H_{\exp }=0.11\right)$, followed by isolates from peppermint $\left(H_{\mathrm{exp}}=0.16\right)$ and isolates from scotch spearmint $\left(H_{\exp }=0.67\right)$.

Plots of the mean number of genotypes expected in relation to the number of loci scored reached saturation, indicating that a sufficient number of loci were analyzed (Fig. 1). Overall, genotypic richness $\left(g_{\text {obs }}\right)$ was greater in the potato population $\left(g_{\text {obs }}=\right.$ $18)$ compared with the population from mint $\left(g_{\text {obs }}=10\right)$ (Table 2$)$. When corrected for the smallest sample size $(n=20)$, estimated genotypic richness was greater among populations from potato $\left(g_{\exp }=6.0\right)$ compared with mint $\left(g_{\exp }=3.2\right)$. The population consisting of isolates sampled from other hosts exhibited the highest $g_{\text {obs }}\left(g_{\text {obs }}=18\right)$ even when corrected for the smallest sample size $\left(g_{\exp }=10.2\right)$, whereas the population from field soils exhibited $g_{\text {obs }}$ and $g_{\text {exp }}$ values of 7 for both uncorrected and corrected sample sizes. The multilocus haplotypes of isolates characterized in this study are summarized in Table 1.

Potato and mint populations exhibited relatively lower indices of evenness compared with the populations from other hosts and field soils (Table 2). A single haplotype, designated H04, was associated with potato in Idaho, Montana, North Dakota, Nebraska, Oregon, South Dakota, Washington, and Wyoming. This haplotype accounted for $70 \%$ of isolates from potato. Haplotype H04 was also isolated from $V$. dahliae-infected peppermint and sugar beet (Beta sp.) in Idaho; maple (Acer sp.), raspberry, blackberry (Rubus spp.), and watermelon (Citrullus sp.) in Oregon; and cherry (Prunus sp.) in Washington, as well as from field soils in Ohio, Oregon, and Washington. All isolates designated as haplotype $\mathrm{H} 04$ belonged to VCG4A, with the exception of two isolates which were characterized as VCG4B and one isolate which belonged to VCG4A/B. Haplotypes H06, H07, and H08 were found to contain isolates belonging to both VCG4A and VCG4B subgroups. Isolates characterized as H09 were found to belong to VCG4A, VCG4B, and VCG4A/B subgroups. In all, $88 \%$ of mint isolates were represented by a single VCG2B haplotype (H02). Haplotype H02 was sampled from mint in Indiana, Oregon, Michigan, Montana, and several counties in Washington. This haplotype was also recovered from maple in Oregon, sugar beet in Idaho, and skullcap (Scutellaria sp.) in Washington. All $\mathrm{H} 02$ haplotypes belonged to VCG2B, with the exception of a single isolate from native spearmint which was characterized as VCG2A/B. One haplotype (H30) was sampled from peppermint in Michigan and Scotch spearmint in Washington. Indices of evenness were greatest and similar among populations consisting of isolates from other hosts and field soils (Table 2).

Overall, indices of genotypic diversity $\left(H^{\prime}, N_{1}\right.$, and $\left.G\right)$ were significantly greater among populations associated with potato 
compared with mint (Table 2). The reference populations consisting of isolates obtained from other hosts and field soils exhibited relatively high and moderate indices of genotypic diversity, respectively (Table 2).

Genetic differentiation between populations from mint and potato. PCoA was used to identify clusters of isolates based on genetic similarity. Mint and potato haplotypes clustered into distinct genetic groups (Fig. 2). Haplotypes from potato could be further subdivided into two groups. The first potato group consisted entirely of haplotypes characterized as VCG4A. The second group consisted mostly of haplotypes belonging to VCG4B intermixed with haplotypes belonging to VCG4A and VCG4A/B. As indicated above, some haplotypes were represented by multiple isolates that were determined to belong to different VCG4 subgroups (Table 1). VCG2A/B isolates from potato and watermelon clustered with VCG2B isolates from mint but VCG2A/B isolates from pistachio and tomato clustered more closely with VCG4B isolates from potato. Isolates belonging to VCG1 and VCG3 were separate but clustered more closely with isolates from potato characterized as VCG4A. Minimum spanning networks of haplotypes from mint and haplotypes from potato were distinctly different (Fig. 3). Haplotype H02 was located at the center of the mint network, with several closely related haplotypes diverging from the predominant clone with no evidence of reticulation. However, reticulation was observed in the minimum spanning network among haplotypes scored as VCG4B and VCG4A/B

Pairwise comparisons of Slatkin's $R_{\mathrm{st}}$ and AMOVA were used to test the hypothesis that $V$. dahliae populations from mint and potato were not genetically differentiated. Isolates from infected potato plants, infected certified seed tubers, infested tare soil associated with certified seed lots, and field soils associated with commercial potato production were not significantly different $\left(R_{\mathrm{st}} \leq 0.12\right)$ (Table 4). Mint and potato populations were significantly different from each other using pairwise comparisons of Slatkin's $R_{\text {st }}\left(R_{\mathrm{st}}=0.70\right)$ (Table 5$)$. The population from mint was also significantly different from field soil $\left(R_{\mathrm{st}}=0.58\right)$ but was not significantly different from isolates from other hosts $\left(R_{\mathrm{st}}=0.11\right)$. In all, $>94 \%$ of the observed genetic variability could be explained by genetic differentiation between mint and potato popu-

TABLE 2. Number of Verticillium dahliae isolates sampled; number of haplotypes observed; Nei's expected heterozygosity; and indices of genotypic richness, evenness, and diversity ${ }^{\mathrm{a}}$

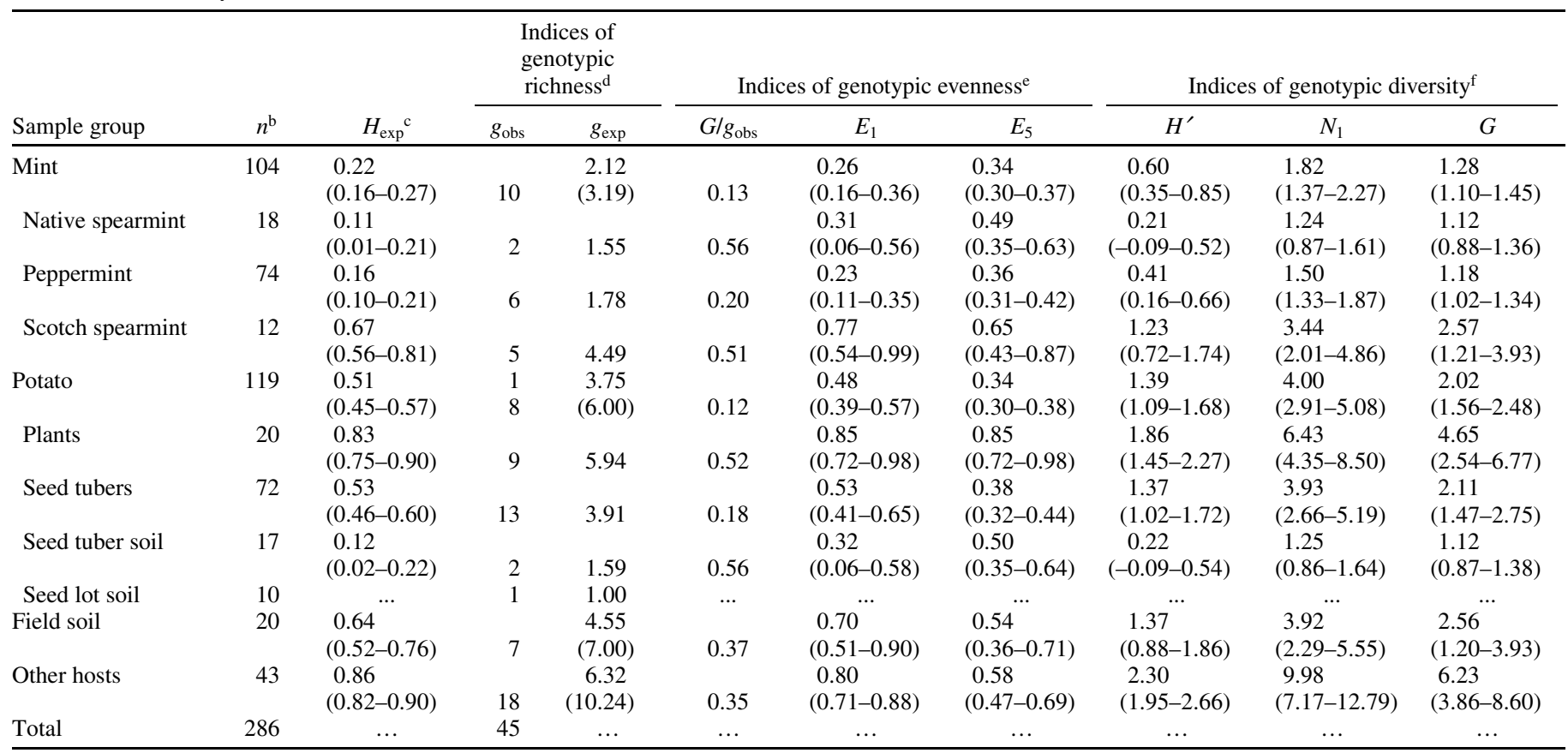

a Values in parenthesis represent $95 \%$ confidence intervals calculated using the SAS macro <jackboot.sas $>$ and 2,000 bootstrap replicates.

${ }^{\mathrm{b}}$ Number of individuals.

${ }^{\mathrm{c}} H_{\text {exp }}$, Nei's expected heterozygosity calculated using Arlequin version 3.5.

${ }^{\mathrm{d}}$ Genotypic richness indices: $g_{\text {obs }}$, number of multilocus haplotypes observed; $g_{\text {exp }}$, number of multilocus haplotypes expected for a sample size of 10 isolates and 20 isolates (parentheses), calculated using GenoDive version 1.1 and 10,000 jackknife replicates.

e Genotypic evenness indices: $E_{1}=H^{\prime}\left[\left[\ln \left(g_{\max }\right)\right]\right.$, where $g_{\max }$ is the maximum number of expected genotypes; $E_{5}=\left[(G-1) /\left(N_{1}-1\right)\right]$

${ }^{\mathrm{f}}$ Genotypic diversity indices: $H^{\prime}=$ Shannon-Wiener diversity index; $N_{1}=e^{\mathrm{H}^{\prime}}$; and $G=$ Stoddart-Taylor genotypic diversity index.

TABLE 3. Nei's expected heterozygosity $\left(H_{\text {exp }}\right)$ estimated for each microsatellite locus and Verticillium dahliae sample group ${ }^{\mathrm{a}}$

\begin{tabular}{|c|c|c|c|c|c|c|c|c|c|c|c|}
\hline \multirow[b]{2}{*}{ Group } & \multicolumn{10}{|c|}{ Locus } & \multirow[b]{2}{*}{ Mean $^{\mathrm{b}}$} \\
\hline & VD1 & VD2 & VD3 & VD4 & VD96 & VD8 & VD10 & VD26 & VD27 & VD74 & \\
\hline Mint & $\begin{array}{c}0.06 \\
(0.36)\end{array}$ & $\begin{array}{c}0.08 \\
(0.51)\end{array}$ & $\begin{array}{c}0.04 \\
(0.36)\end{array}$ & $\begin{array}{c}0.06 \\
(0.51)\end{array}$ & $\begin{array}{c}0.06 \\
(0.38)\end{array}$ & $\begin{array}{c}0.04 \\
(0.38)\end{array}$ & $\begin{array}{c}0.15 \\
(0.76)\end{array}$ & $\begin{array}{c}0.02 \\
(0.20)\end{array}$ & $\begin{array}{c}0.02 \\
(0.20)\end{array}$ & $\begin{array}{c}0.02 \\
(0.20)\end{array}$ & $\begin{array}{c}0.05 \pm 0.04 \\
(0.38 \pm 0.17)\end{array}$ \\
\hline Field soil & $\begin{array}{c}0.61 \\
(0.81)\end{array}$ & $\begin{array}{c}0.62 \\
(0.81)\end{array}$ & $\begin{array}{c}0.19 \\
(0.29)\end{array}$ & $\begin{array}{c}0.57 \\
(0.52)\end{array}$ & $\begin{array}{c}0.61 \\
(0.81)\end{array}$ & $\begin{array}{c}0.19 \\
(0.29)\end{array}$ & $\begin{array}{c}0.19 \\
(0.29)\end{array}$ & $\begin{array}{c}0.19 \\
(0.29)\end{array}$ & $\begin{array}{c}0.42 \\
(0.67)\end{array}$ & $\begin{array}{c}0.57 \\
(0.52)\end{array}$ & $\begin{array}{c}0.41 \pm 0.20 \\
(0.53 \pm 0.23)\end{array}$ \\
\hline
\end{tabular}

${ }^{\text {a }}$ Results in parentheses represent analyses conducted using clone-corrected data.

${ }^{\mathrm{b}}$ Mean and standard deviation. 
lations using AMOVA, and this difference was statistically significant $(P=0.03)$ (Table 6$)$. When clone-corrected data were employed in AMOVA, $>76 \%$ of the overall genetic variation was due to differences between mint and potato sample groups, and this was also significant $(P=0.03)$. AMOVA was also used to test the hypothesis that $V$. dahliae isolates belonging to VCG4A and VCG4B were not genetically differentiated. Although genetic

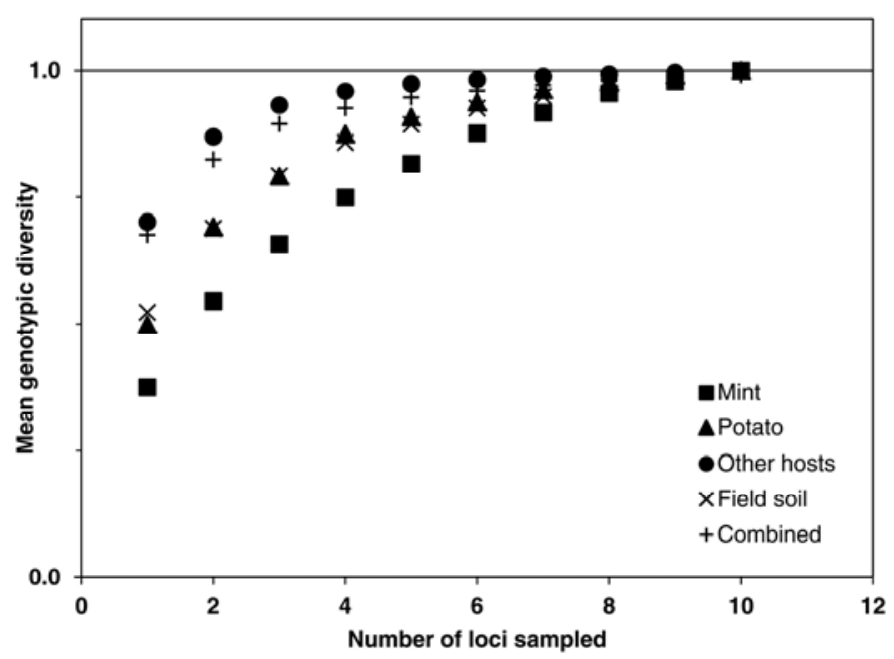

Fig. 1. Plots of mean genotypic diversity versus the number of loci sampled among Verticillium dahliae populations from mint, potato, other hosts, field soils, and all four sample groups combined. differences between VCG4A and VCG4B accounted for $73 \%$ of the total variation, VCG4 subgroups from potato were not significantly different $(P=0.34)$ (Table 7$)$. Only $20 \%$ of the overall genetic variability was due to differences in VCG4 subgroups when clone-corrected data ere used for AMOVA $(P=0.33)$.

Private alleles and migration rate estimates. When scaled for sample size $(n=9)$, VCG2B isolates obtained from mint possessed $\approx 1.70 \pm 0.37$ private alleles per locus compared with $0.30 \pm 0.15$ and $0.22 \pm 0.14$ private alleles among VCG4A and VCG4B isolates associated with potato, respectively (Table 8). Estimates of mutation-scaled population sizes and migration rates were conducted even though several populations comprised $<10$ haplotypes after clone correction. Migration was detected from VCG4B to VCG4A subgroups but not the converse or between VCG2B and either VCG4 subgroup (Table 8). More than two migrants per generation from tare soil to potato were estimated but not vice versa, and more than two migrants per generation were detected between field soils and potato (Table 9). More than one migrant per generation was estimated between infected seed tubers and plants.

Aggressiveness assays. The two positive control isolates used in the aggressiveness assays, isolate 111 from mint and isolate 653 from potato, were characterized as $\mathrm{H} 02$ and $\mathrm{H} 04$ haplotypes, respectively. Only five isolates caused Verticillium wilt symptoms on mint that were significantly different than asymptomatic waterinoculated controls. The mint-associated haplotype H02 caused significantly greater Verticillium wilt symptoms on mint $(P \leq$ 0.05 ) in both trials, even when the haplotype was sampled from other hosts such as maple and sugar beet (Fig. 4). Both VCG2 and VCG4A isolates from watermelon caused significant Verticillium

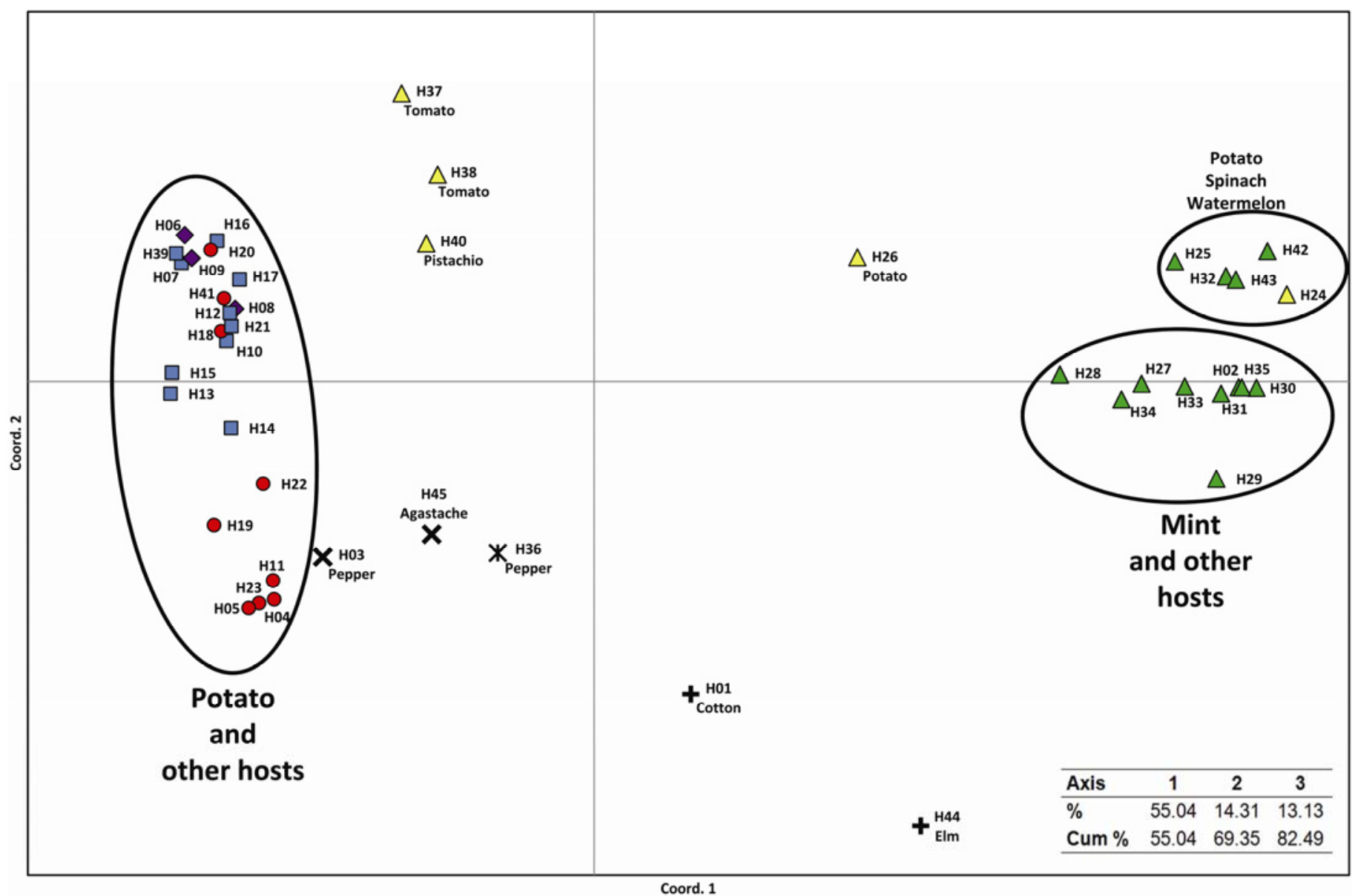

Fig. 2. Principal coordinate analysis of Verticillium dahliae microsatellite haplotypes from different hosts and vegetative compatibility groups (VCGs). Haplotypes correspond to those listed in Table 1. Vegetative compatibility groups are represented by the following: black cross = VCG1; yellow triangle = VCG2 and VCG2A; green triangle $=\mathrm{VCG} 2 \mathrm{~B}$; black $\mathrm{X}=\mathrm{VCG} 3$; red circle $=\mathrm{VCG} 4 \mathrm{~A}$; blue square $=\mathrm{VCG} 4 \mathrm{~B}$; purple diamond $=\mathrm{VCG} 4 \mathrm{~A} / \mathrm{B}$; and black star $=$ not determined . 
wilt symptoms on mint in both trials $(P \leq 0.05)$. In all, 9 of 19 isolates caused significant $(P \leq 0.05)$ Verticillium wilt symptoms in potato. The potato-associated haplotype $\mathrm{H} 04$ isolated from potato, maple, sugar beet, and tare soil caused significantly greater Verticillium wilt symptoms on potato compared with controls in both trials. In addition, VCG4B isolates from spinach and strawberry and VCG2 isolates from pistachio and tomato caused significantly greater disease symptoms on potato $(P \leq 0.05)$. In general, most isolates were able to cause moderate to severe disease on potato and only mild to moderate symptoms on mint (Fig. 4).

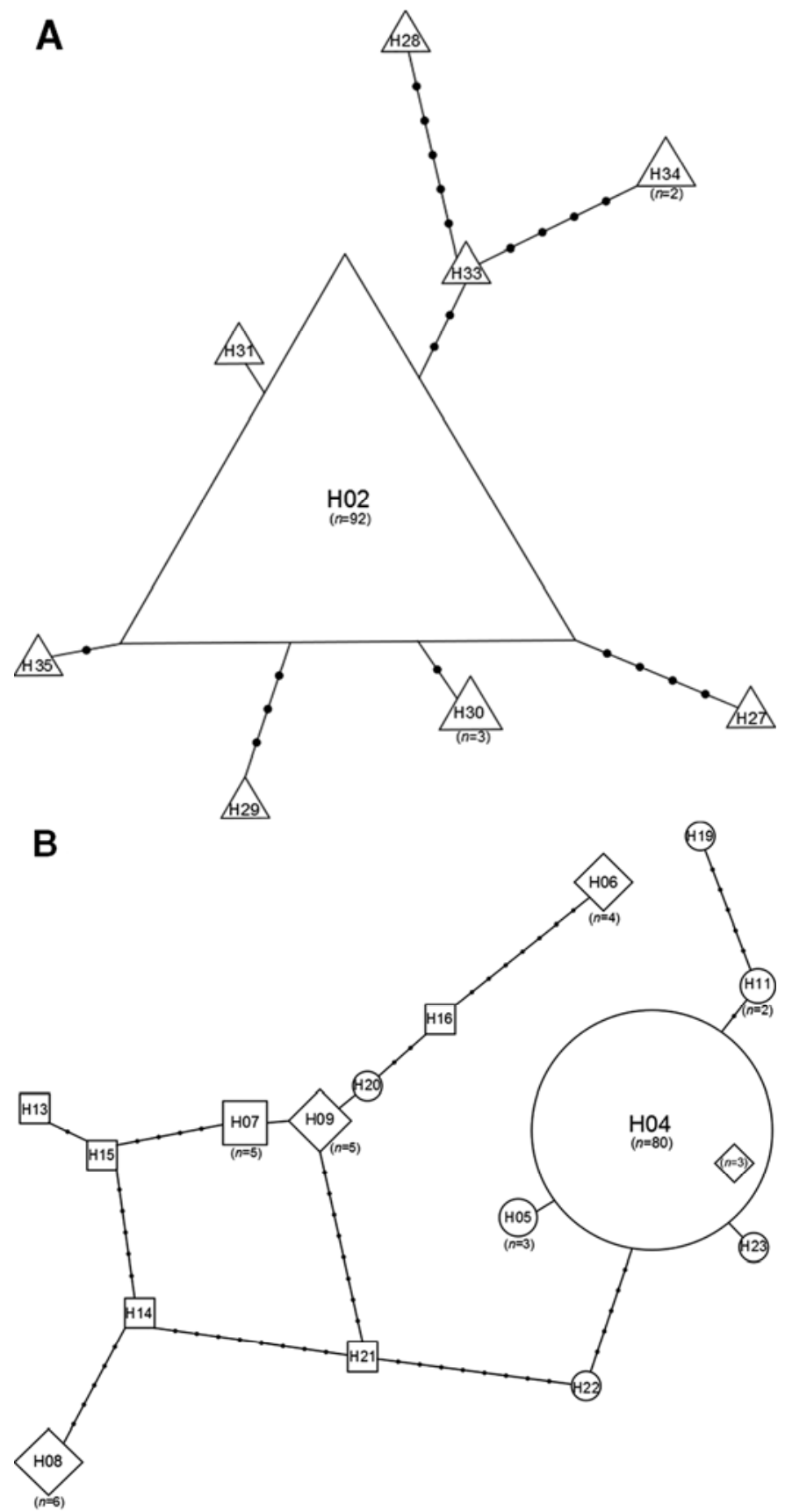

Fig. 3. Minimum spanning networks of A, Verticillium dahliae haplotypes from mint belonging to vegetative compatibility group (VCG)2B (triangles) and $\mathbf{B}, V$. dahliae haplotypes from potato belonging to VCG4A (circles), VCG4B (squares), and VCG4A/B (diamonds). Distances between haplotypes are based on genetic distances calculated using the stepwise mutation model. Sizes of shapes are scaled according the haplotype frequencies. Black dots represent one mutation step. Haplotype codes refer to multilocus haplotypes as listed in Table 1.

\section{DISCUSSION}

In total, $286 \mathrm{~V}$. dahliae isolates associated with mint, potato, and other hosts were analyzed for mating type, VCG, and using 10 microsatellite markers previously shown to be polymorphic in populations from California and Wisconsin (3). V. dahliae populations from mint and potato appear to fit a clonal reproductive model, with all isolates being a single mating type and multiple occurrences of multilocus haplotypes over time and space. The mint and potato populations each consisted of a different predominant multilocus haplotype. This is in contrast with a previous study using isolates from California and Wisconsin, in which most isolates were assigned to a unique haplotype and recombination was detected. The different results obtained in this study may be due to the fewer number of microsatellites markers used, since the use of more markers increases the probability of assigning isolates to unique haplotypes, or may be a result of differences in crops and cropping systems between the Columbia Basin and coastal California.

The predominant mint and potato haplotypes were primarily assigned to vegetative compatibility groups VCG2B and VCG4A, respectively, and aggressiveness assays demonstrated that these haplotypes frequently caused severe disease on their respective hosts, even when isolated from a different host. However, haplotype, VCG, and aggressiveness did not appear to be linked in this study, because several haplotypes were characterized as different VCGs or exhibited differences in aggressiveness on mint and potato. Significant genetic differentiation was observed between $V$. dahliae populations from mint and potato, and gene flow estimates, together with the existence of private alleles, indicated restricted gene flow between the two populations. Mint populations appeared to be the most genetically divergent in this study and contained more than twice the number of private alleles as the potato and field soil sample groups despite its low relative genotypic diversity and evenness. Several factors likely contribute to the genetic differentiation of $V$. dahliae from potato and mint, including the occurrence of a single mating type, as well as differences in VCG and host adaptation in $V$. dahliae populations from these hosts. The genetic structure observed may also be a due to a historical geographic separation of hosts and their associ-

TABLE 4. Pairwise comparisons of Slatkin's $R_{\mathrm{ST}}$ values for Verticillium dahliae sampling groups from infected potato plants, infected certified seed tubers, infested tare soil from certified seed lots, and field soils associated with commercial potato production $^{\mathrm{a}}$

\begin{tabular}{lccc}
\hline Sample group & Plants & Seed tuber & Tare soil \\
\hline Plants & $\ldots$ & $\ldots$ & $\ldots$ \\
Seed tubers & 0.12021 & $\ldots$ & $\ldots$ \\
Tare soil & -0.05572 & 0.03815 & $\ldots$ \\
Field soil & -0.08772 & 0.10495 & -0.05655 \\
\hline
\end{tabular}

a An $R_{\mathrm{ST}}$ value of 0 indicates no separation, $0<R_{\mathrm{ST}}<0.05$ indicates negligible differentiation, $0.05 \leq R_{\mathrm{ST}}<0.25$ indicates moderate differentiation, $0.25 \leq$ $R_{\mathrm{ST}}<1$ indicates high differentiation, and $R_{\mathrm{ST}}=1$ indicates complete differentiation.

TABLE 5. Pairwise comparisons of Verticillium dahliae populations from different hosts and sources using Slatkin's $R_{\mathrm{ST}}{ }^{\mathrm{a}}$

\begin{tabular}{lccc}
\hline Sample group & Mint & Potato & Field soil \\
\hline Mint & $\ldots$ & $\ldots$ & $\ldots$ \\
Potato & $0.69616^{\mathrm{b}}$ & $\ldots$ & $\ldots$ \\
Field soil & $0.57953^{\mathrm{b}}$ & -0.01858 & $\ldots$ \\
Other hosts & 0.10721 & 0.16990 & 0.04527 \\
\hline
\end{tabular}

a An $R_{\mathrm{ST}}$ value of 0 indicates no separation, $0<R_{\mathrm{ST}}<0.05$ indicates negligible differentiation, $0.05 \leq R_{\mathrm{ST}}<0.25$ indicates moderate differentiation, $0.25 \leq$ $R_{\mathrm{ST}}<1$ indicates high differentiation, and $R_{\mathrm{ST}}=1$ indicates complete differentiation.

b Significant at $P<0.0083(0.05 / 6)$. 
ated pathogen populations although, currently, both crops can be grown in close proximity or even in rotation with each other in many U.S. states.

Although not statistically significant, mint populations had lower gene diversity than populations from potato. The greater diversity of $V$. dahliae populations associated with potato in North America may be due to the fact that potato is grown in more states and on more hectares compared with mint. In addition, large-scale potato production has been occurring over a longer period of time in North America compared with mint, which may have led to greater diversity over time. As expected, the population of isolates from other hosts that was used as the outgroup exhibited the greatest amount of diversity and evenness, which was likely an artifact of the diverse sampling of hosts, VCGs, and geography. The predominant mint and potato haplotypes were represented among isolates collected from other hosts, indicating the potential for these haplotypes to infect hosts other than mint and potato.

Previous studies demonstrated that VCG2B is the predominant VCG isolated from mint. In this study, the most common haplo- type from mint, H02, was consistently characterized from VCG2B isolates and accounted for $88 \%$ of the mint isolates sampled. This haplotype was sampled from mint in several mint-producing states (Indiana, Oregon, Michigan, Montana, and several counties in Washington) and in samples collected more than a decade apart, indicating that this haplotype is well established in commercial mint production. Several closely related haplotypes appear to be mutational derivatives of this haplotype, as indicated by the minimum spanning network. Overall, these results indicate that a single haplotype is common and widespread among isolates of $V$. dahliae in mint, consistent with previous studies concluding that a host-adapted pathotype of $V$. dahliae is present in mint production $(11,30)$. Mint is vegetatively propagated and the potential exists for $V$. dahliae populations to persist clonally in this host. In addition, mint is a perennial crop which may contribute to the annual increase of host-adapted genotypes in fields over time. Although propagative materials for mint production start as disease-free tissue-culture or greenhouse-grown plants, rhizomes used for propagative materials are typically increased in

TABLE 6. Analysis of molecular variance of Verticillium dahliae grouped by host of isolation ${ }^{\mathrm{a}}$

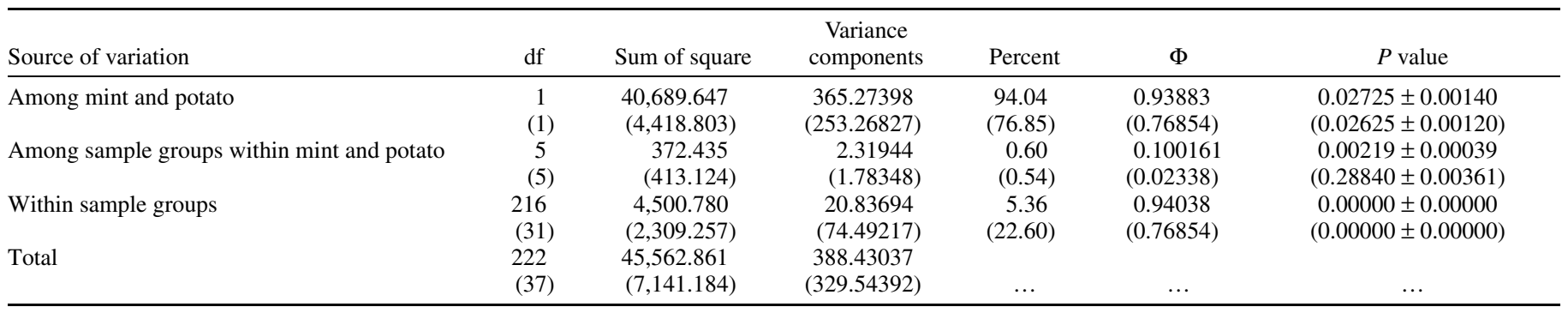

${ }^{a}$ Results in parentheses represent analyses conducted using clone-corrected data.

TABLE 7. Analysis of molecular variance of Verticillium dahliae within and among vegetative compatibility group (VCG) 4 subgroups (VCG4A and VCG4B) ${ }^{\mathrm{a}}$

\begin{tabular}{|c|c|c|c|c|c|c|}
\hline Source of variation & df & Sum of square & $\begin{array}{c}\text { Variance } \\
\text { components }\end{array}$ & Percent & $\Phi$ & $P$ value \\
\hline Among VCG4 subgroups & $\begin{array}{c}1 \\
(1)\end{array}$ & $\begin{array}{r}1,213.012 \\
(136.668)\end{array}$ & $\begin{array}{l}39.25300 \\
(8.27648)\end{array}$ & $\begin{array}{c}73.22 \\
(19.99)\end{array}$ & $\begin{array}{c}0.73221 \\
(0.19985)\end{array}$ & $\begin{array}{c}0.33777 \pm 0.00373 \\
(0.33046 \pm 0.00396)\end{array}$ \\
\hline Among sample groups within VCG4 subgroups & $\begin{array}{c}2 \\
(2)\end{array}$ & $\begin{array}{c}78.578 \\
(77.894)\end{array}$ & $\begin{array}{r}2.22744 \\
(1.5097)\end{array}$ & $\begin{array}{c}4.16 \\
(3.65)\end{array}$ & $\begin{array}{c}0.15516 \\
(0.04556)\end{array}$ & $\begin{array}{c}0.06455 \pm 0.00185 \\
(0.32346 \pm 0.00354)\end{array}$ \\
\hline Within sample groups & $\begin{array}{c}81 \\
(19)\end{array}$ & $\begin{array}{c}982.386 \\
(600.917)\end{array}$ & $\begin{array}{c}12.12822 \\
(31.62719)\end{array}$ & $\begin{array}{c}22.62 \\
(76.37)\end{array}$ & $\begin{array}{c}0.77376 \\
(0.23630)\end{array}$ & $\begin{array}{c}0.00000 \pm 0.00000 \\
(0.05137 \pm 0.00177)\end{array}$ \\
\hline Total & $\begin{array}{c}84 \\
(22)\end{array}$ & $\begin{array}{r}2,273.976 \\
(815.478)\end{array}$ & $\begin{array}{c}53.60866 \\
(41.41338)\end{array}$ & $\ldots$ & $\ldots$ & . \\
\hline
\end{tabular}

${ }^{\text {a }}$ Results in parentheses are from analyses conducted using clone-corrected data.

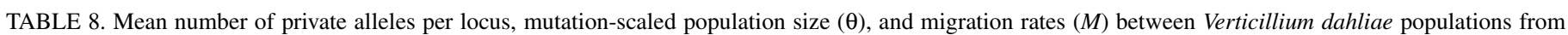
mint and potato belonging to different vegetative compatibility groups (VCGs)

\begin{tabular}{|c|c|c|c|c|c|}
\hline Sample group & Private alleles/locus ${ }^{\mathrm{a}}$ & $\theta^{\mathrm{b}}$ & $\operatorname{Mint}(\mathrm{VCG} 2 \mathrm{~B})^{\mathrm{b}}$ & Potato (VCG4A) ${ }^{\mathrm{b}}$ & Potato (VCG4B) ${ }^{\mathrm{b}}$ \\
\hline Mint (VCG2B) & $1.70(1.33-2.07)$ & 1.3917 (1.0859-1.8324) & & $0.1291(0.0657-0.2239)$ & $0.0222(0.0037-0.0693)$ \\
\hline Potato (VCG4A) & $0.30(0.15-0.45)$ & $0.6402(0.4324-0.8757)$ & $0.2298(0.0883-0.4762)$ & & $1.9675(1.4458-2.6521)$ \\
\hline Potato (VCG4B) & $0.22(0.08-0.36)$ & $1.0832(0.8788-1.3797)$ & $0.0387(0.0067-0.1082)$ & $0.5398(0.3612-0.7691)$ & $\ldots$ \\
\hline
\end{tabular}

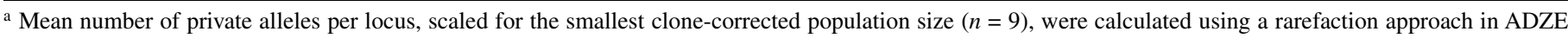
1.0 .

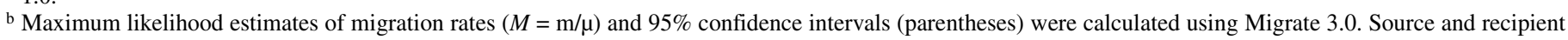
populations are in columns and rows, respectively.

TABLE 9. Mutation-scaled population size $(\theta)$ and migration rates $(M)$ between Verticillium dahliae isolates from infected potato plants, infected certified seed tubers, infested tare soil from certified seed lots, and field soils associated with commercial potato production ${ }^{\mathrm{a}}$

\begin{tabular}{lccccc}
\hline Sample group & $\theta$ & Plants & Seed tubers & Tare soil & Field soil \\
\hline Plants & $1.0603(0.8095-1.3946)$ & $\ldots$ & $1.5393(1.1330-2.0345)$ & $2.5252(1.9924-3.1608)$ & $2.6831(2.1248-3.3909)$ \\
Seed tubers & $0.8750(0.7233-1.0700)$ & $1.0682(0.7343-1.4480)$ & $\ldots$ & $1.4131(1.0724-1.8203)$ & $0.4439(0.2662-0.6870)$ \\
Tare soil & $0.6211(0.4925-0.8004)$ & $0.7812(0.5322-1.1328)$ & $0.7851(0.5475-1.0823)$ & $\ldots$ & $1.1273(0.8333-1.4902)$ \\
Field soil & $0.8838(0.6568-1.1792)$ & $3.0168(2.3669-3.7000)$ & $0.3766(0.1749-0.6564)$ & $1.2333(0.8925-1.6498)$ & $\ldots$ \\
\hline
\end{tabular}

${ }^{\text {a }}$ Maximum likelihood estimates of migration rates $(M=\mathrm{m} / \mu)$ and $95 \%$ confidence intervals (parentheses) were calculated using Migrate 3.0. Source and recipient populations are in columns and rows, respectively. 
the field prior to planting for commercial production, which can result in the introduction of $V$. dahliae into planting stock. Although many mint rootstock certification standards express a zero tolerance for Verticillium wilt in planting stock, there are acknowledged risks associated with undetected $V$. dahliae in planting materials, especially in those grown under less strict land requirements. Therefore, it is important that field increases of propagative materials for commercial production occur in soils free of $V$. dahliae, and especially in soils free of haplotypes known to be aggressive on mint.

The predominant haplotype associated with potato was H04 and this haplotype was most often characterized as VCG4A.
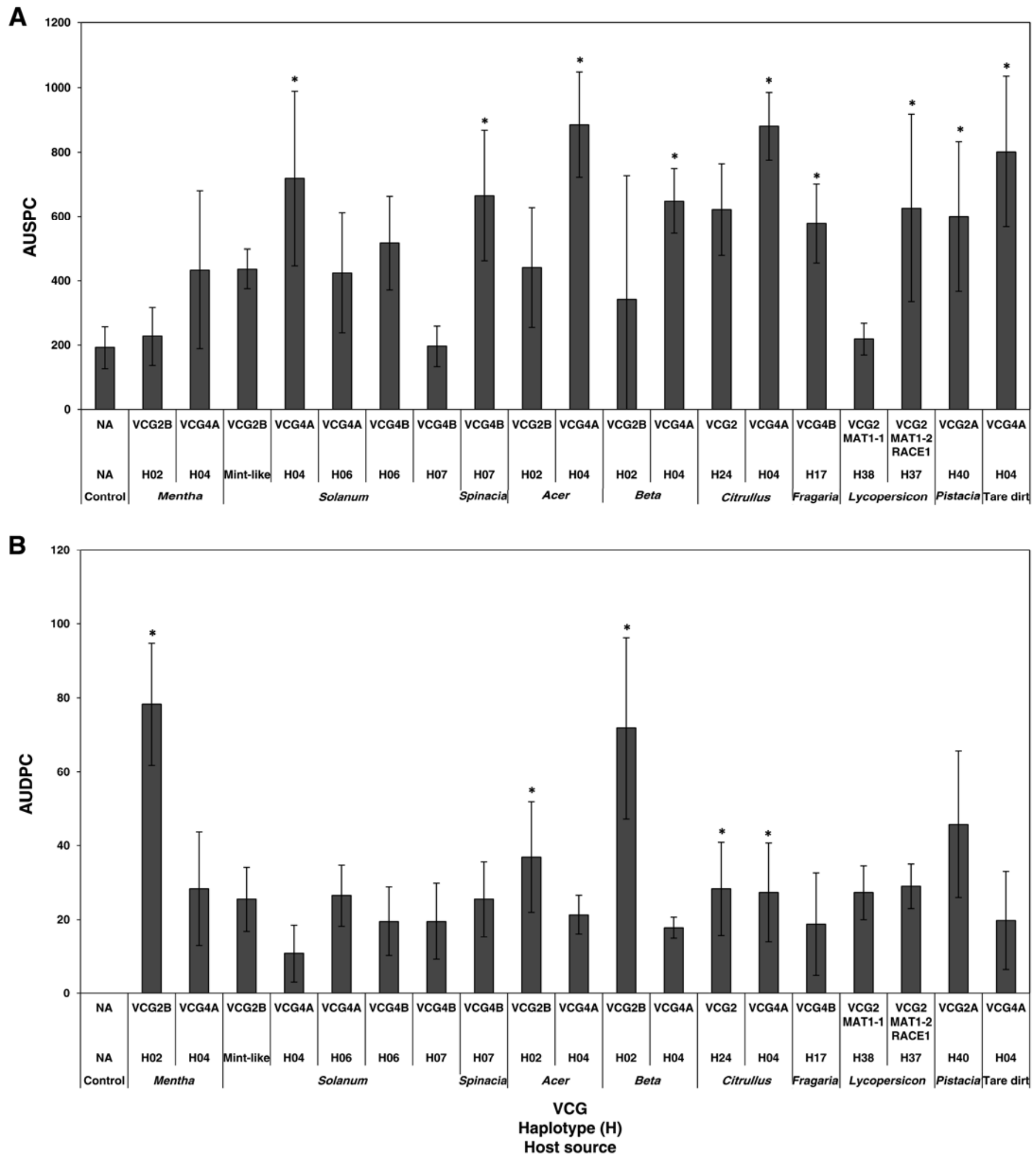

Fig. 4. A, Area under senescence progress curve (AUSPC) values of 'Russet Norkotah' potato and B, area under disease progress curve (AUDPC) values of Scotch spearmint following inoculations with different haplotypes of Verticillium dahliae. Haplotypes of $V$. dahliae were collected from different hosts and were characterized according to vegetative compatibility groups (VCGs). Asterisks indicate a significant difference $(P \leq 0.05)$ from water-inoculated control treatments using Dunnett's test. 
Haplotype H04 appears to be common in North American commercial potato production, having been observed in samples collected from seven states and over a period of several decades, from before 1983 through 2010. This haplotype was also isolated from mint, indicating the potential for this potato-associated haplotype to infect mint. In addition, one isolate from watermelon which was characterized as $\mathrm{H} 04$ caused significant disease in spearmint and many isolates caused mild symptoms; therefore, growers should use caution when rotating between potato and mint. Haplotype H04 was the predominant haplotype associated with infected seed tubers, tare soils associated with certified potato seed lots, and field soils associated with commercial potato production. Seed tubers, which are used to vegetatively propagate potato for commercial planting, are produced in 15 states of the United States and shipped to production areas throughout North America and the world. In this study, many isolates from infected seed tubers and infested soil associated with certified seed tubers were haplotype H04. It should be noted that $65 \%$ of seed lots sampled originated from Idaho, Montana, and Oregon and many of the isolates characterized as $\mathrm{H} 04$ were from these three states. In addition, the majority of the seed tuber isolates characterized as haplotype H04 was collected between 2007 and 2011. However, $>39 \%$ of seed tuber-producing hectares are located in Idaho, Montana, and Oregon and these results suggest that a clonal population of $V$. dahliae is widespread in seed tubers used for commercial planting. Haplotype H04 was also found in infected seed tubers produced in several states prior to 1997, demonstrating its association with potato for more than a decade. Nonetheless, isolates from other seed-producing areas were typically haplotypes other than $\mathrm{H} 04$, indicating that genetic variation exists in $V$. dahliae populations among seed tuber-producing states. Like mint, potato propagative materials are started as tissue-culture or greenhouse-grown plants but are increased in fields over several seasons, a necessary practice but one which can result in the annual increase of pathogens in later generations of seed tubers. Higher estimated migration rates were observed between field soil and plants and between tare soil and plants compared with seed tubers and plants, which is in agreement with previous studies concluding that field and tare soils are important sources of inoculum for Verticillium wilt of potato (13-15). However, the potential does exist for infected seed tubers to infest field soils and infect plants in subsequent seasons rather than in the current season. Infected seed tubers or infested soil associated with seed tubers may provide a means for the movement of $V$. dahliae and facilitate the migration of novel or host-adapted genotypes across long distances.

PCoA separated VCG1, VCG2B, and VCG4 isolates into distinct groups. However, two clusters of VCG2 isolates were observed using PCoA. The first group consisted of isolates from tomato (Lycopersicon esculentum) and pistachio (Pistacia vera) and was more closely related to the VCG4 group, while the other group consisted of isolates from potato and watermelon and was more closely related to the mint VCG2B group. These results support a previous study suggesting that two genetically distinct groups of VCG2 isolates exist within $V$. dahliae, with one lineage exhibiting a closer genetic relationship with VCG4B (9). VCG3 isolates clustered more closely with VCG4 isolates and moderate complementation was observed between one VCG3 isolate from Agastache spp. and VCG4A tester strains, suggesting that these two VCGs are genetically similar. Complementation between VCG3 and VCG4A tester strains in V. dahliae has been previously reported (40).

VCG4 subgroups from potato and seed potato were not significantly differentiated using AMOVA and migration estimates indicated that VCG4B contributes nearly two migrants per generation into VCG4A. A cluster of VCG4 isolates, consisting of VCG4A, VCG4B, and VCG4A/B isolates, was observed using PCoA in this study, indicating that VCG4A and VCG4B sub- groups are not genetically isolated. The lack of differentiation among VCG4A and VCG4B isolates may be due to gene flow or a relatively recent divergence. Some VCG4 isolates from potato exhibited strong complementation with both VCG4 subgroups and were designated as VCG4A/B; therefore, the possibility exists for gene flow to occur between VCG4A and VCG4B isolates via hyphal anastomosis with VCG4A/B isolates. In addition, several VCG4 isolates associated with potato also exhibited weak complementation with VCG4A or VCG4B, which may also facilitate gene flow between these subgroups over time. These results suggest that vegetative incompatibility may not present an absolute barrier to gene flow among VCG4A and VCG4B subgroups.

All isolates assayed for mating type in this study possessed the MAT1-2 idiomorph, with the exception of isolate 461 from tomato, which possessed the MAT1-1 idiomorph. These results suggest that the potential for sexual reproduction is low in $V$. dahliae populations impacting mint and potato in North America. The MATl-2 idiomorph was found to be predominant in V. dahliae populations in a previous study, occurring in 143 of 154 isolates from various hosts (22). The MAT1-1 idiomorph has been found in isolates from tomato collected in California and Brazil, as well as isolates from other solanaceous hosts such as potato and pepper. The MAT1-2 idiomorph was also found to be prevalent in $V$. dahliae populations from Japan, though the MAT1-1 idiomorph was found in isolates from tomato and a few other isolates from other hosts and countries (43).

A greater number of sampled isolates caused moderate to severe Verticillium wilt symptoms on potato compared with mint. The H04 haplotype, which was predominant in potato and most often characterized as VCG4A, generally caused the most severe symptoms on potato regardless of host origin, followed by haplotypes belonging to VCG2 and VCG4B. These results are consistent with previous work demonstrating a continuum of aggressiveness among $V$. dahliae isolates on potato (39). In contrast, the H02 mint haplotype caused severe symptoms on mint whereas other haplotypes and VCGs were generally not as aggressive on mint. A number of isolates characterized as haplotype H02 in this study were shown to be aggressive on different Mentha spp. and hybrids in the current and previous studies $(11,16)$. This is in agreement with previous studies concluding that isolates from mint are highly aggressive on mint and cause Verticillium wilt symptoms on a limited range of hosts $(11,16,30,35)$. Host selection, or interactions between host resistance and pathogen aggressiveness, may result in differences among $V$. dahliae populations in their abilities to colonize certain hosts, impacting the genetic diversity of pathogen populations found in different crops and field. A greater susceptibility to a wider range of $V$. dahliae isolates may increase the genotypic variation observed in $V$. dahliae populations sampled from potato plants compared with mint due to less host-selection pressure.

We hypothesized that differences in vegetative compatibility, aggressiveness, or mating type compatibility between $V$. dahliae populations from mint and potato would result in a reduction in gene flow and genetic and genotypic differentiation between pathogen populations affecting these two hosts. Populations of the pathogen from mint and potato were genetically differentiated, indicating that there is limited migration or gene flow between them. Differences in vegetative compatibility and the occurrence of only a single mating type likely contribute to genetic drift and differentiation among $V$. dahliae populations from mint and potato. Populations of $V$. dahliae in mint and potato were characterized by the presence of highly aggressive, clonally reproducing haplotypes, and these haplotypes are widely distributed and established in commercial mint and potato production. Pathogen dissemination via infected propagative materials may lead to the establishment and predominance of certain host-adapted strains, especially in vegetatively propagated hosts. In addition, the long survival of $V$. dahliae as microsclerotia in soils may also result in 
the continued persistence of certain host-adapted pathotypes. This, coupled with limited gene flow among unlike VCGs and the lack of sexual reproduction, may contribute to the persistence and differentiation of host-adapted $V$. dahliae populations in some cropping systems.

\section{ACKNOWLEDGMENTS}

PPNS Number 0608, Department of Plant Pathology, College of Agricultural, Human, and Natural Resource Sciences Agricultural Research Center, Project Number WNP00678, Washington State University, Pullman 99164-6430. We thank the Washington State Potato Commission and the Mint Industry Research Council for financial and in-kind support of this project, colleagues who provided some of the isolates used in this study, D. R. See for providing the technology and assistance required for fragment analysis, and J. A. L. Johnson for technical assistance.

\section{LITERATURE CITED}

1. Agapow, P. M., and Burt, A. 2001. Indices of multilocus linkage disequilibrium. Mol. Ecol. Notes 1:101-102.

2. Atallah, Z. K., Maruthachalam, K., Davis, R. M., Klosterman, S. J., and Subbarao, K. V. 2009. Characterization of 22 highly polymorphic microsatellite loci in the cosmopolitan fungal plant pathogen Verticillium dahliae. Mol. Ecol. Resour. 9:1460-1559.

3. Atallah, Z. K., Maruthachalam, K., Toit, L. d., Koike, S. T., Michael Davis, R., Klosterman, S. J., Hayes, R. J., and Subbarao, K. V. 2010. Population analyses of the vascular plant pathogen Verticillium dahliae detect recombination and transcontinental gene flow. Fungal Genet. Biol. 47:416-422.

4. Balloux, F., and Lugon-Moulin, N. 2002. The estimation of population differentiation with microsatellite markers. Mol. Ecol. 11:155-165.

5. Beerli, P., and Felsenstein, J. 1999. Maximum-likelihood estimation of migration rates and effective population numbers in two populations using a coalescent approach. Genetics 152:763-773.

6. Beerli, P., and Felsenstein, J. 2001. Maximum likelihood estimation of a migration matrix and effective population sizes in $n$ subpopulations by using a coalescent approach. Proc. Natl. Acad. Sci. USA 98:4563-4568.

7. Berbegal, M., Ortega, A., Jiménez-Gasco, M. M., Olivares-García, C., Jiménez-Díaz, R. M., and Armengol, J. 2010. Genetic diversity and host range of Verticillium dahliae isolates from artichoke and other vegetable crops in Spain. Plant Dis. 94:396-404.

8. Bhat, R. G., and Subbarao, K. V. 1999. Host range specificity in Verticillium dahliae. Phytopathology 89:1218-1225.

9. Collado-Romero, M., Mercado-Blanco, J., Olivares-García, C., and Jiménez-Díaz, R. M. 2008. Phylogenetic analysis of Verticillium dahliae vegetative compatibility groups. Phytopathology 98:1019-1028.

10. Dobinson, K. F., Harrington, M. A., Omer, M., and Rowe, R. C. 2000. Molecular characterization of vegetative compatibility group 4A and 4B isolates of Verticillium dahliae associated with potato early dying. Plant Dis. 84:1241-1245.

11. Douhan, L. I., and Johnson, D. A. 2001. Vegetative compatibility and pathogenicity of Verticillium dahliae from spearmint and peppermint. Plant Dis. 85:297-302.

12. Dung, J. K. S., du Toit, L. J., and Johnson, D. A. 2011. Verticillium wilt of skullcap and potential for pathogen dissemination via seeds and stems. Plant Dis. 95:1147-1152.

13. Dung, J. K. S., Hamm, P. O., Eggers, J. E., and Johnson, D. A. 2012. Incidence and impact of Verticillium dahliae in soil associated with certified potato seed lots. Phytopathology 103:55-63.

14. Dung, J. K. S., Ingram, J. T., Cummings, T. F., and Johnson, D. A. 2012. Impact of seed lot infection on the development of black dot and Verticillium wilt of potato in Washington. Plant Dis. 96:1179-1184.

15. Dung, J. K. S., and Johnson, D. A. 2012. Roles of infected seed tubers and soilborne inoculum on Verticillium wilt of 'Russet Burbank' potato. Plant Dis. 96:379-383.

16. Dung, J. K. S., Schroeder, B. K., and Johnson, D. A. 2010. Evaluation of Verticillium wilt resistance in Mentha arvensis and M. longifolia genotypes. Plant Dis. 94:1255-1260.

17. Dunnett, C. W. 1955. A multiple comparisons procedure for comparing several treatments with a control. J. Am. Stat. Assoc. 509:1096-1121.
18. Estoup, A., Jarne, P., and Cornuet, J.-M. 2002. Homoplasy and mutation model at microsatellite loci and their consequences for population genetics analysis. Mol. Ecol. 11:1591-1604.

19. Excoffier, L., Laval, G., and Schneider, S. 2005. Arlequin (version 3.0): An integrated software package for population genetics data analysis. Evol. Bioinf. Online 1:47-50.

20. Excoffier, L., and Smouse, P. E. 1994. Using allele frequencies and geographic subdivision to reconstruct gene trees within a species: Molecular variance parsimony. Genetics 136:343-359.

21. Grünwald, N. J., Goodwin, S. B., Milgroom, M. G., and Fry, W. E. 2003. Analysis of genotypic diversity data for populations of microorganisms. Phytopathology 93:738-746.

22. Inderbitzin, P., Davis, R. M., Bostock, R. M., and Subbarao, K. V. 2011. The ascomycete Verticillium longisporum is a hybrid and a plant pathogen with an expanded host range. PLoS ONE 6:e18260.

23. Joaquim, T. R., and Rowe, R. C. 1990. Reassessment of vegetative compatibility relationships among strains of Verticillium dahliae using nitratenonutilizing mutants. Phytopathology 80:1160-1166.

24. Joaquim, T. R., and Rowe, R. C. 1991. Vegetative compatibility and virulence of strains of Verticillium dahliae from soil and potato plants. Phytopathology 81:552-558.

25. Johnson, D. A. 2009. Transmission of Phytophthora infestans from infected potato seed tubers to emerged shoots. Plant Dis. 94:18-23.

26. Katan, T. 2000. Vegetative compatibility in populations of Verticilliuman overview. Pages 69-86 in: Advances in Verticillium: Research and Disease Management. E. C. Tjamos, R. C. Rowe, J. B. Heale, and D. R. Fravel, eds. American Phytopathological Society Press, St. Paul, MN.

27. Leslie, J. F. 1993. Fungal vegetative compatibility. Annu. Rev. Phytopathol. 31:127-150.

28. Meirmans, P. G., and Van Tienderen, P. H. 2004. GENOTYPE and GENODIVE: Two programs for the analysis of genetic diversity of asexual organisms. Mol. Ecol. Notes 4:792-794.

29. Nei, M. 1973. Analysis of gene diversity in subdivided populations. Proc. Natl. Acad. Sci. USA 70:3321-3323.

30. Nelson, R. 1947. The specific pathogenesis of the Verticillium that causes wilt of peppermint. (Abstr.) Phytopathology 37:17.

31. Ohta, T., and Kimura, M. 1973. A model of mutation appropriate to estimate the number of electrophoretically detectable alleles in a finite population. Genet. Res. 22:201-204.

32. Omer, M. A., Johnson, D. A., Douhan, L. I., Hamm, P. B., and Rowe, R. C. 2008. Detection, quantification, and vegetative compatibility of Verticillium dahliae in potato and mint production soils in the Columbia Basin of Oregon and Washington. Plant Dis. 92:1127-1131.

33. Omer, M. A., Johnson, D. A., and Rowe, R. C. 2000. Recovery of Verticillium dahliae from North American certified seed potatoes and characterization of strains by vegetative compatibility and aggressiveness. Am. J. Potato Res. 77:325-331.

34. Peakall, R. O. D., and Smouse, P. E. 2006. GENALEX 6: Genetic analysis in Excel. Population genetic software for teaching and research. Mol. Ecol. Notes 6:288-295.

35. Qin, Q. M., Vallad, G. E., Wu, B. M., and Subbarao, K. V. 2006. Phylogenetic analyses of phytopathogenic isolates of Verticillium spp. Phytopathology 96:582-592.

36. Rice, W. R. 1989. Analyzing tables of statistical tests. Evolution 43:223225.

37. Schuelke, M. 2000. An economic method for the fluorescent labeling of PCR fragments. Nat. Biol. 18:233-234.

38. Slatkin, M. 1995. A measure of population subdivision based on microsatellite allele frequencies. Genetics 139:457-462.

39. Strausbaugh, C. A. 1993. Assessment of vegetative compatibility and virulence of Verticillium dahliae isolates from Idaho potatoes and tester strains. Phytopathology 83:1253-1258.

40. Strausbaugh, C. A., Schroth, M. N., Weinhold, A. R., and Hancock, J. G. 1992. Assessment of vegetative compatibility of Verticillium dahliae tester strains and isolates from California potatoes. Phytopathology 82:6168.

41. Szpiech, Z. A., Jakobsson, M., and Rosenberg, N. A. 2008. ADZE: A rarefaction approach for counting alleles private to combinations of populations. Bioinformatics 24:2498-2504.

42. Teacher, A. G. F., and Griffiths, D. J. 2010. HapStar: Automated haplotype network layout and visualization. Mol. Ecol. Resour. 11:151153.

43. Usami, T., Itoh, M., and Amemiya, Y. 2009. Asexual fungus Verticillium dahliae is potentially heterothallic. J. Gen. Plant Pathol. 75:422-427. 